\title{
Double-resonant nanostructured gold surface for multiplexed detection
}

\author{
Antonio Minopoli, ${ }^{1,2}$ Emanuela Scardapane, ${ }^{1}$ Bartolomeo Della Ventura, ${ }^{1}$ Julian A. Tanner, ${ }^{3}$ Andreas \\ Offenhäusser, ${ }^{2}$ Dirk Mayer, ${ }^{2, *}$ and Raffaele Velotta ${ }^{1, *}$ \\ ${ }^{1}$ Department of Physics “E. Pancini”, University Federico II, Via Cintia 26, 80126 Naples, Italy. \\ ${ }^{2}$ Institute of Biological Information Processing (IBI-3), Bioelectronics, Forschungszentrum Jülich, 52425 Jülich, \\ Germany. \\ ${ }^{3}$ School of Biomedical Sciences, University of Hong Kong, Hong Kong SAR, China. \\ *Corresponding authors: rvelotta@unina.it, dirk.mayer@fz-juelich.de
}

\section{Table of contents}

Section S1. Analysis of scanning electron micrographs.

Section S2. Coding of numerical simulations.

Section S3. Optical response of homogeneously sized gold nanoparticles.

Section S4. Substrate biofunctionalization.

Section S5. Fluorescence picture acquisition, processing and analysis.

Section S6. Analysis of the bright spots.

Section S7. Off-resonance simulations.

Section S8. Analysis of the electric field.

Section S9. Electromagnetic response of the substrate with perfectly spherical nanoparticles.

Section S10. Electromagnetic response of non-spherical nanoparticles.

Section S11. Fluorescence intensity in non-PEF conditions.

Section S12. Chemicals and materials.

Section S13. Photochemical immobilization technique.

Section S14. Microfluidic system.

Section S15. Preparation of buffer solutions.

References. 


\section{Section S1. Analysis of scanning electron micrographs.}

SEM images were acquired by Zeiss LEO 1550VP field emission scanning electron microscope with a nominal resolution of $1 \mathrm{~nm}$ at $20 \mathrm{kV}$ acceleration voltage by collecting secondary electrons with an In-Lens detector. The morphology of the substrate was analysed processing the micrographs by ImageJ software. Firstly, objects were isolated from the background by thresholding the raw image (Figures $\mathrm{S} 1 \mathrm{a}$ and $\mathrm{S} 1 \mathrm{~b}$ ), then adjacent nanoparticles were segmented by watershedding (Figure S1c). "Analyze Particles" tool implemented in ImageJ was run to retrieve information about the object perimeter $p$, area $S$, shape descriptors, and centroid coordinates. Figure S1d shows an example of processed micrograph in which the nanoparticles are decomposed in outline and inner area. Afterwards, nanoparticle diameter was estimated as $D=2 \sqrt{S / \pi}$, whereas the centre-to-centre distance distribution was carried out by calculating the distance of each centroid from its nearest neighbours.

(a)

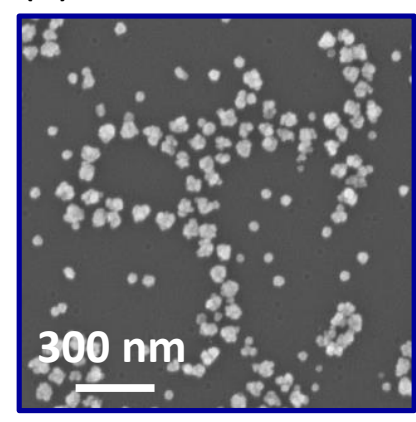

(b)

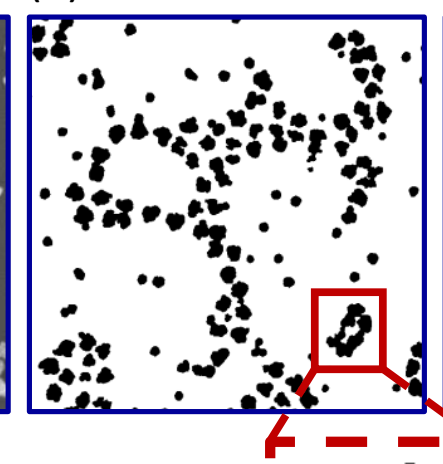

(c)

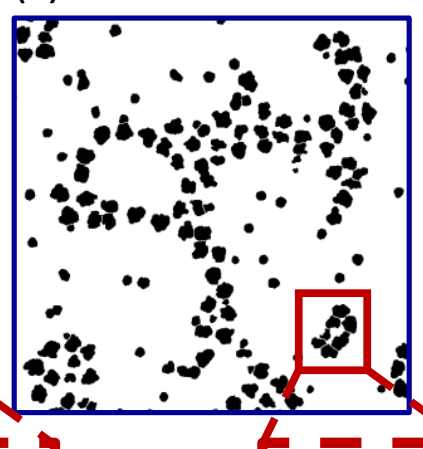

(d)

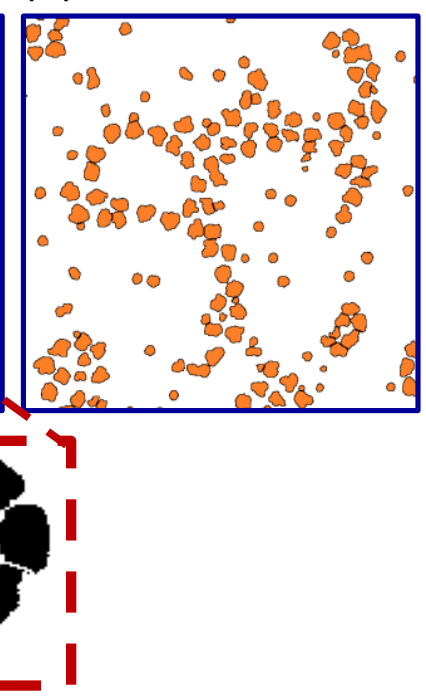

Figure S1. (a) Example of raw SEM micrograph at high magnification. Corresponding (b) thresholded and (c) segmented image. The insets show a magnification of a nanoparticle cluster highlighting the capability to effectively segment adjacent objects by the watershedding algorithm. (d) Processed image in which the nanoparticles are decomposed in outlines (black line) and inner region (orange filling). 


\section{Section S2. Coding of numerical simulations.}

The optical response of the nanostructured substrate was simulated by using the finitedifference time-domain (FDTD) method implemented in "FDTD solutions" tool of Lumerical software. The Maxwell's equations are numerically solved in the time domain within a Mie problem-like framework by discretizing the space over a mesh and evaluating the evolution of the electric and magnetic fields in each cell.

\section{Section S2.A. Branch pattern of spherical AuNPs.}

An illustration of the simulation workspace is shown in Figure S2a. The optical behaviour of the substrate was explored through linearly polarized waves (400-800 nm wavelength, 0-180 polarization angle with angular step of $15^{\circ}$ ) propagating along $z$ direction. A photodetector placed in the $x-y$ plane was dedicated to measure the electric field over time $\left(1.25 \times 1.25 \mu \mathrm{m}^{2}\right.$ size) (not shown in Figure S2a), whereas a second photodetector positioned on the opposite side of the workspace was set to collect the transmitted photons to work out the extinction spectrum of the nanostructure (400-800 $\mathrm{nm}$ wavelength, $1 \mathrm{~nm}$ spectral resolution). The nanostructure was realized by positioning homogeneous gold spheres ${ }^{1}$ onto a layer of silicon dioxide $^{2}$ (200 $\mathrm{nm}$ thickness) according to the real morphology provided by SEM images. Nanoparticle centroids and diameters were retrieved by raw SEM images as described in Section S1. Then, homogeneous gold spheres were shaped in the Lumerical environment using "addsphere" script command. The refractive index of surrounding environment was set equal to 1 .

Periodic boundary conditions (BCs) were set along $x$ and $y$ directions to extend the simulation over an infinite array. Bloch BCs were used for simulations with non-zero polarization angles in order to compensate the phase shift arising when an electromagnetic disturbance with a non-zero angle should be reinjected at the opposite workspace site. Perfect matched layer BCs along $z$ direction (steep angle profile, 12 layers) warranted the complete absorption of the wave backscattered through the light source and that traveling beyond the photodetector. ${ }^{3}$ The spatial resolution of the mesh was set equal to $1 \mathrm{~nm}$ to warrant high accuracy of the results while holding the simulation time within few hours.

\section{Section S2.B. Branch pattern of rough AuNPs.}

Technical parameters of simulations were set as in the Section S2.A. In addition, we implemented a degree of roughness onto the surface of spheres. Firstly, homogenous gold spheres were placed onto a layer of silicon dioxide ${ }^{2}$ (200 nm thickness) according to the real 
morphology. The diameters were reduced of $20 \mathrm{~nm}$ as compared to the real size (see Section S1). Secondly, gold hemispheres of radius $10 \mathrm{~nm}$ were randomly arranged onto the spherical surfaces allowing interpenetration. More precisely, 20 (40) (80) (120) (160) (200) gold hemispheres were placed onto spheres whose real diameter lied in the range 10-20 nm (20-30 $\mathrm{nm})(30-40 \mathrm{~nm})(40-60 \mathrm{~nm})(60-80 \mathrm{~nm})(>80 \mathrm{~nm})$. The size of the resulting rough spheres as well as their degree of roughness was comparable with the real one observed by electron microscopy (Figure S2b).

\section{Section S2.C. Hexagonal cell of non-spherical nanoparticles.}

The optical response of non-spherical nanoparticles was explored through a $x$-polarized wave (300-800 nm wavelength) propagating along $z$ direction. A photodetector placed in the $x-y$ plane was dedicated to measure the electric field over time $\left(160 \times 138 \mathrm{~nm}^{2}\right.$ size $)$, whereas a second photodetector positioned on the opposite side of the workspace was set to collect the transmitted photons to work out the extinction spectrum of the nanostructure $(300-800 \mathrm{~nm}$ wavelength, $1 \mathrm{~nm}$ spectral resolution).

Nanoparticles of different shape (sphere, disk, ring, cube, rhombus, octahedron) and material (copper ${ }^{1}$, silver ${ }^{1}$, gold $^{1}$ ) were arranged in hexagonal cells (80 nm lattice constant) (Figure S2c). Non-spherical nanoparticles were added into Lumerical framework using default 3D models. Also, the response of isolated nanoparticles was studied for comparison. The refractive index of surrounding environment was set equal to 1 . The geometrical parameters and lattice constant of the investigated nanostructures are listed in Table S1.

Symmetric/anti-symmetric BCs set along $x$ and $y$ direction extended the plasmonic response over an infinite 2D array while reduced the simulation time by a factor 8 without worsening the accuracy of the results. Perfect matched layer BCs set in $z$ (steep angle profile, 12 layers) assured perfect absorption of the electromagnetic waves backscattered through the plane containing the light source and incident upon the opposite side of the workspace. ${ }^{3}$ The spatial resolution of the mesh was set equal to $1 \mathrm{~nm}$ to warrant high accuracy of the results while holding the simulation time within few hours. 
(a)

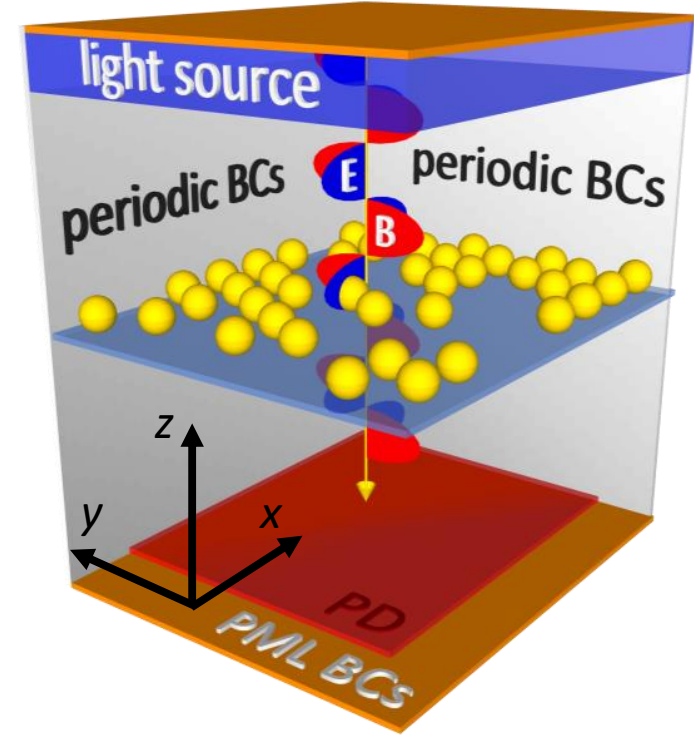

(b)
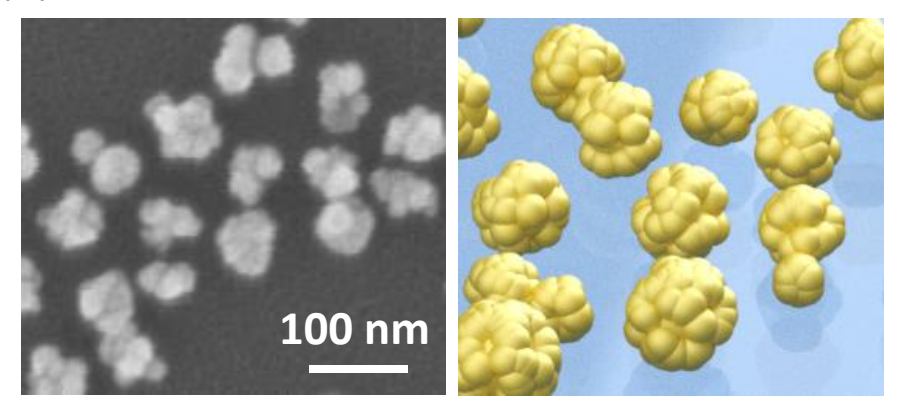

(c)

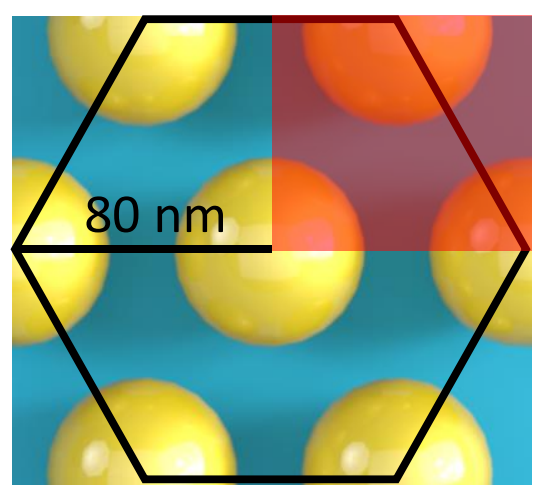

Figure S2. (a) Sketch of the simulation workspace consisting of light source, plasmonic nanostructure, dielectric substrate, photodetector (PD), and appropriate boundary conditions (BCs). (b) Comparison between the real morphology of the substrate observed by SEM (left panel) and a representative model employed in FDTD simulations (right panel). (c) Example of Au spheres arranged in a hexagonal cell. The shaded red rectangle represents the region in which the Maxwell's equations are numerically solved. Then, the solutions are symmetrically/anti-symmetrically extended to the whole hexagonal cell. 
Table S1. Geometrical and lattice parameters of the investigated nanostructures. Dimensions of hexagonally arranged nanoparticles correspond to those of isolated nanoparticles and hence they are omitted to improve the readability.

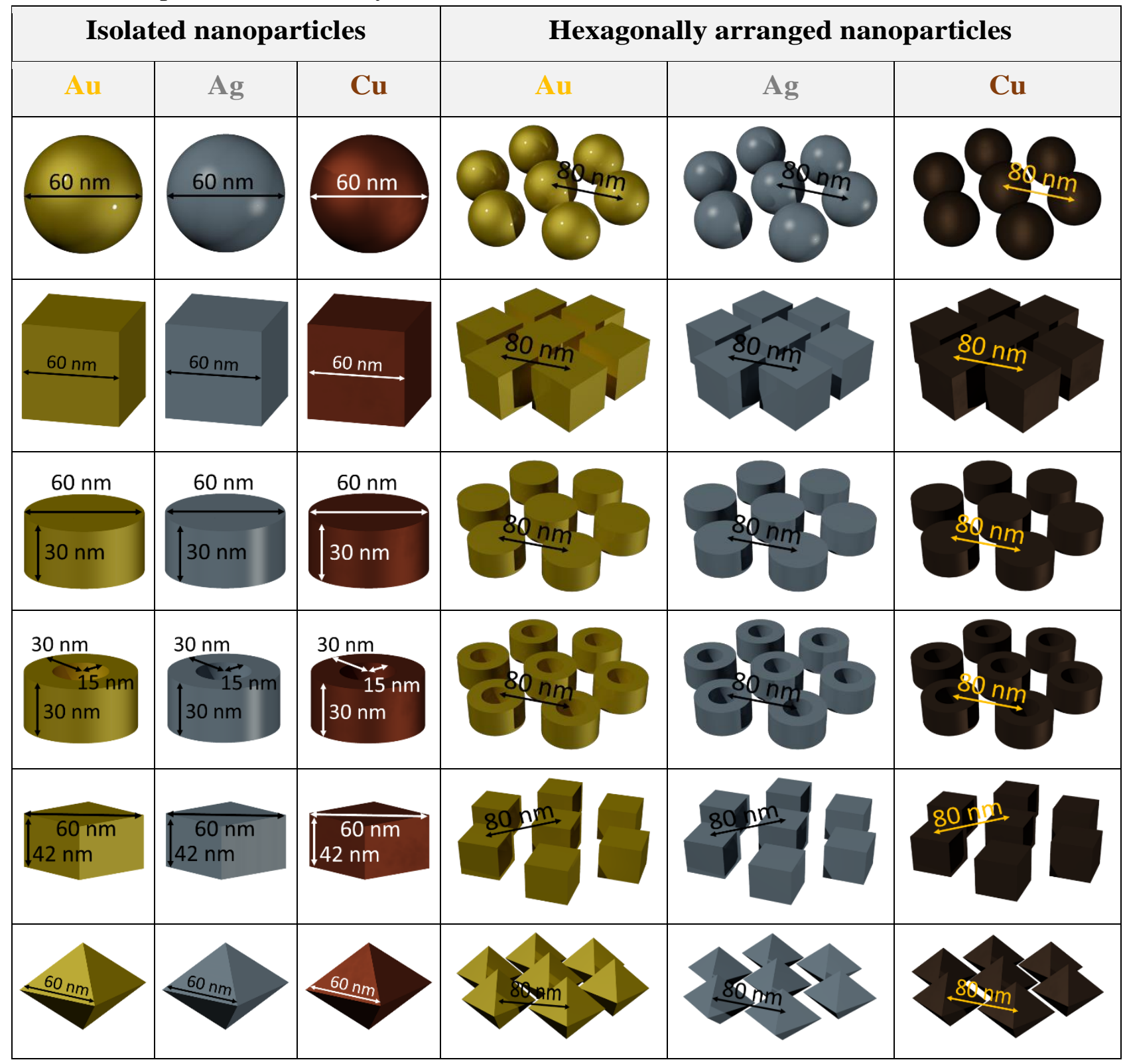




\section{Section S3. Optical response of homogeneously sized gold nanoparticles.}

Numerical simulations worked out with homogeneously sized AuNPs confirmed that the measured double resonance of the real substrate is due to not only the branch architecture, but also the combination of heterogeneously sized AuNPs. Actually, the optical response of a pattern made of 30 $\mathrm{nm}$ diameter AuNPs conveys a broad peak in the extinction spectrum at $540 \mathrm{~nm}$ wavelength. This plasmonic mode may contain both the response of isolated nanoparticles (at $\sim 520 \mathrm{~nm}$ ) and that of plasmon-coupled nanoparticles (Figures S3a, S3b and S3c). On the contrary, the pattern made of 60 $\mathrm{nm}$ diameter AuNPs exhibits two resonances at $540 \mathrm{~nm}$ and $675 \mathrm{~nm}$. Plasmon-uncoupled AuNPs contribute to the first plasmonic mode, whereas plasmon-coupled AuNPs produce the second mode (Figures S3d, S3e and S3f). Note that we coded the script for placing homogeneously sized $60 \mathrm{~nm}$ diameter AuNPs in such a way to avoid particle interpenetration. 
(a)

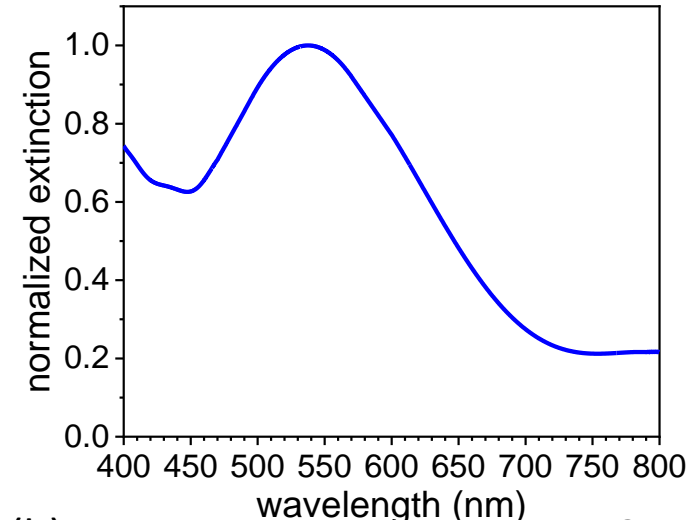

(b)

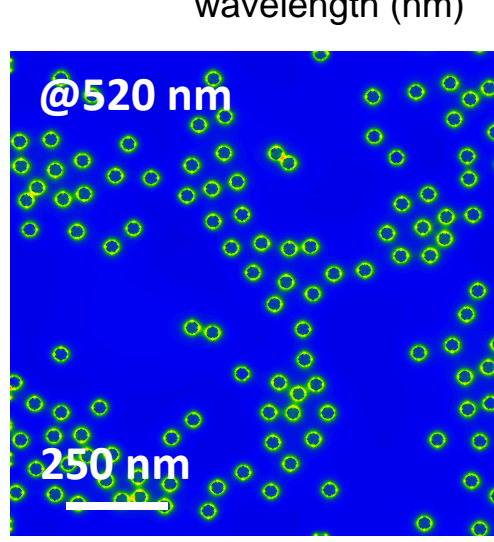

(c)

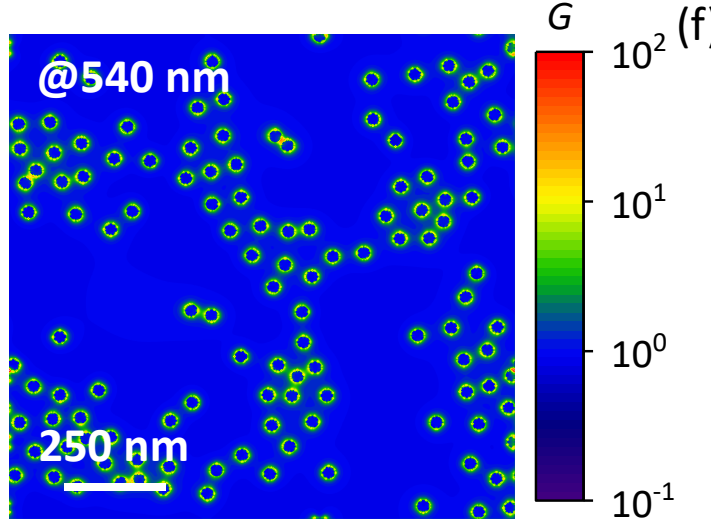

(d)

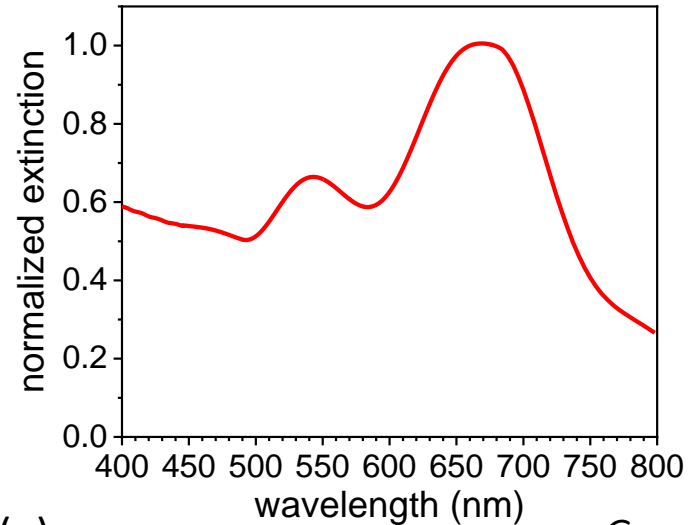

e)
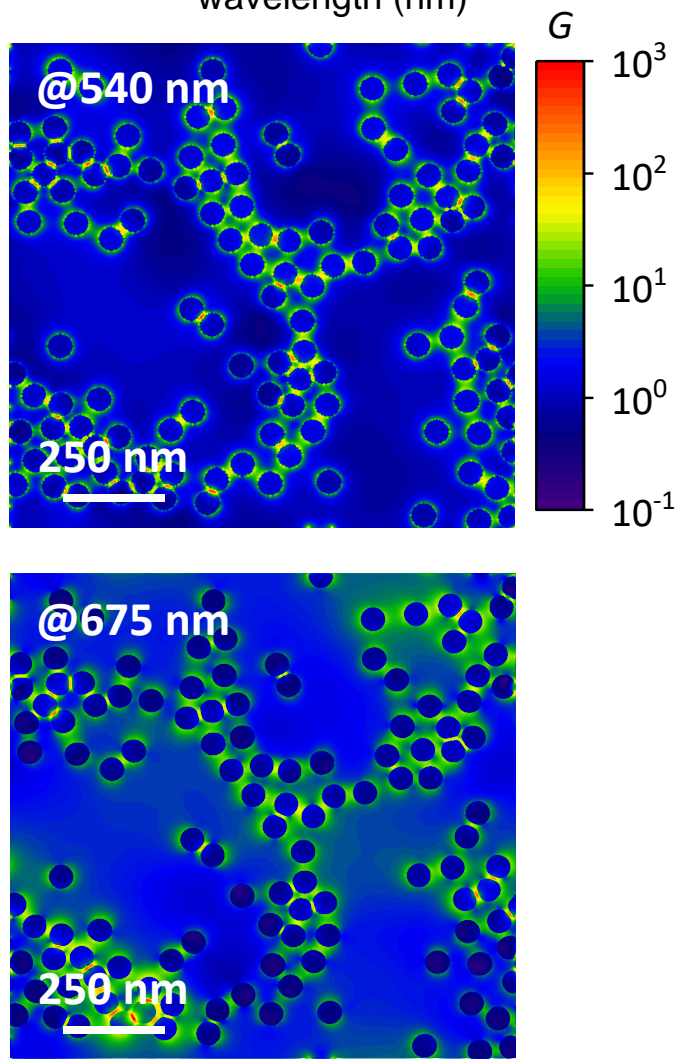

Figure S3. Simulated extinction spectrum of the substrate in the case of homogeneously sized AuNPs of (a) $30 \mathrm{~nm}$ diameter and (d) $60 \mathrm{~nm}$ diameter. Simulated electric field intensity of the substrate worked out at wavelengths (b) $520 \mathrm{~nm}$ and (c) $540 \mathrm{~nm}$ in the case of $30 \mathrm{~nm}$ diameter AuNPs, and (e) $540 \mathrm{~nm}$ and (f) $675 \mathrm{~nm}$ in the case of $60 \mathrm{~nm}$ diameter AuNPs. 
Section S4. Substrate biofunctionalization.

(a)

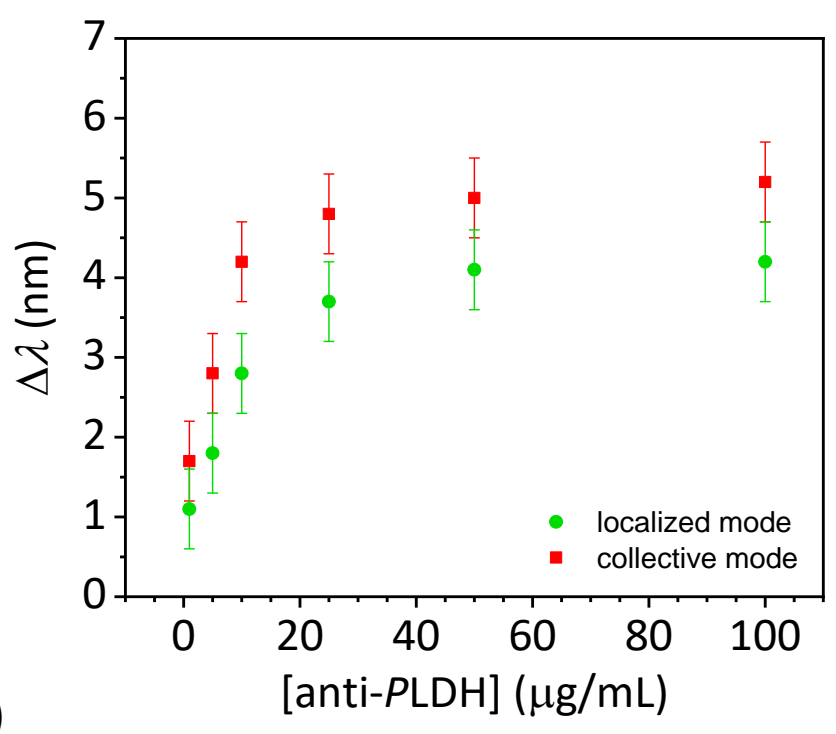

(b)

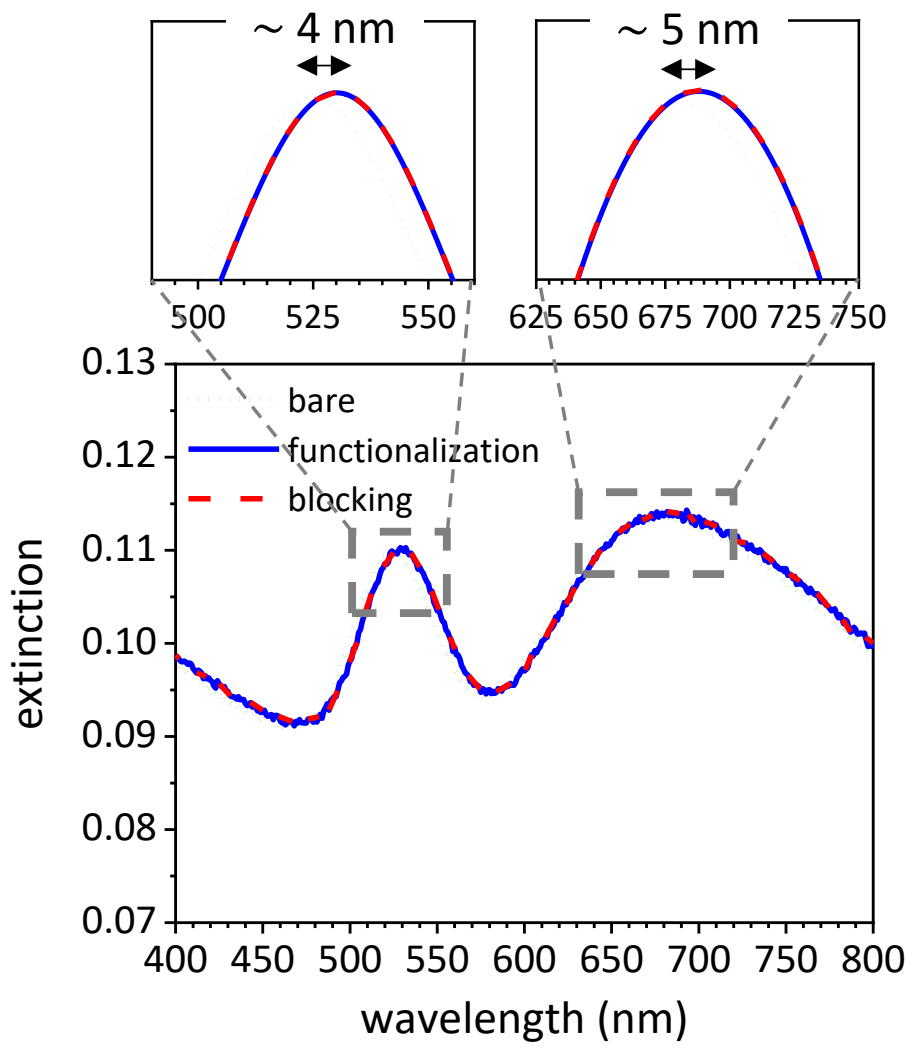

Figure S4. (a) Shift of the plasmon resonance wavelengths $(\Delta \lambda)$ as a function of anti-PLDH concentration for the localized (green circles) and collective (red squares) modes. Both the resonances red-shift as the anti-PLDH concentration increased up to $50 \mu \mathrm{g} / \mathrm{mL}$ that corresponds to a maximum redshift of approximately 4-5 $\mathrm{nm}$. (b) Experimental extinction spectrum of the substrate before (dotted green line) and after functionalization (solid blue line) and blocking (dashed red line). The insets show the Gaussian fits used for evaluating the plasmon resonance wavelength. 


\section{Section S5. Fluorescence picture acquisition, processing and analysis.}

Fluorescence images were acquired by Zeiss Axio Observer Z1 inverted phase contrast fluorescence microscope equipped by Zeiss Colibri.2 LED light source (modules 470 and 625 nm), Zeiss Plan-Apochromat 10x/0.45 Ph1 M27 (FWD = $2.1 \mathrm{~mm}$ ) objective, $38 \mathrm{HE}$ filter (excitation 450-490 nm/emission 500-550nm) and cube 50 Cy5 filter (excitation 625-655 $\mathrm{nm} / \mathrm{emission} 665-715 \mathrm{~nm}$ ), and pco.edge $5.5 \mathrm{sCMOS}$ photodetector (scaling $0.650 \mu \mathrm{m} \times 0.650$ $\mu \mathrm{m}$ per pixel, image size $2560 \times 2160$ pixels, scaled image size $1.66 \mathrm{~mm} \times 1.40 \mathrm{~mm}, 16$ bit dynamic range). The camera exposure time was set to $2 \mathrm{~s}$ for recording every image.

Raw fluorescence images were processed by ImageJ to measure the whole intensity arising from the bright spots. Firstly, RGB images were split into two channels containing the green and red components so to separately analyse the contribution of the two fluorophores used in this work. Since the raw images contained a smooth continuous background (Figure S5a), the rolling ball algorithm was used to flatten any spatial variations of the background by evaluating the average value over a ball around each pixel and then by locally subtracting such a value from the image (Figures S5b and S5c). Note that the fluorescence intensity was scaled by an arbitrary factor to ease the readability of the outcomes.

Figure S5d shows an example of processed image at higher magnification in which green and red channels are merged and the bright spots strikingly emerge from the background, whereas Figures S5e and S5f depict the corresponding intensity histograms of both the channels. Thus, the whole intensity of 5-FAM and Cy5 fluorophores was measured as a function of the spiked PfLDH concentration by summing the signal contribution of every spot.

Aiming at carrying out a reliable and robust analysis, one different substrate was used for each analyte concentration (rather than exploit additive concentrations on the same substrate), and ten fluorescence images were randomly acquired over each sample thereby obtaining an average value of the whole fluorescence intensity \pm standard deviation. 
(a)

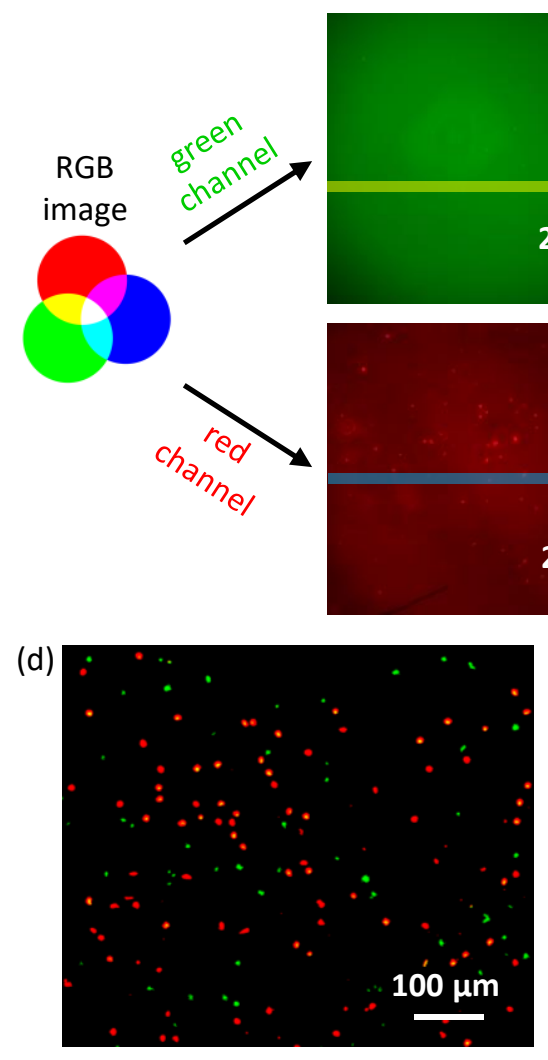

(b)

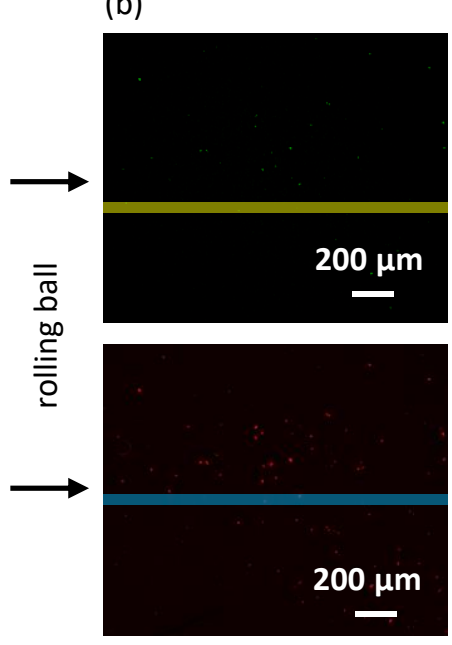

(c)
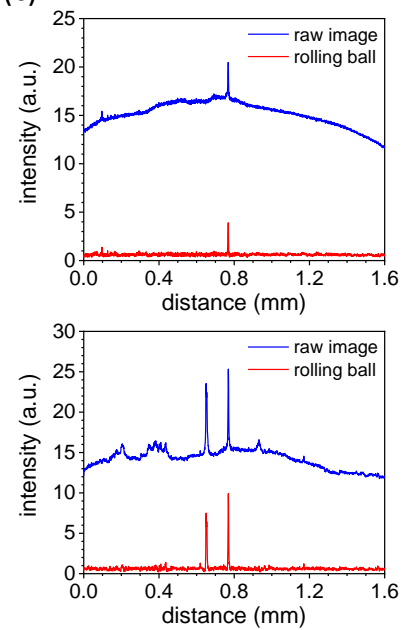
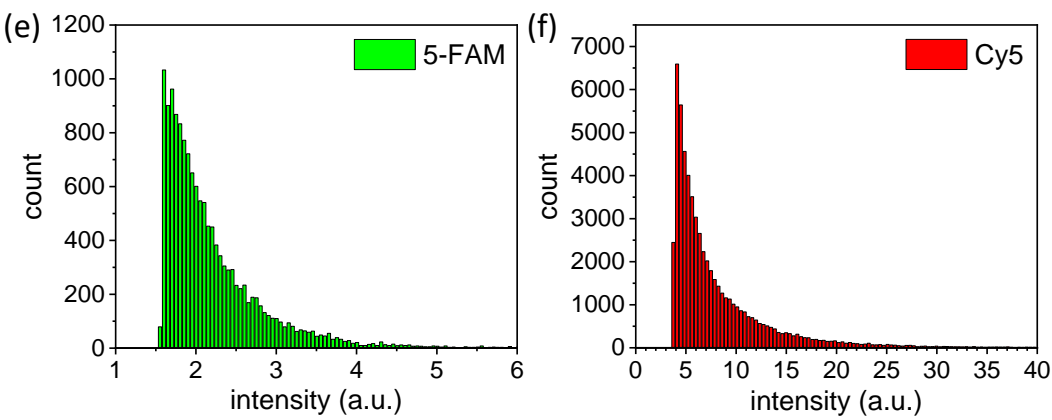

Figure S5. (a) Example of raw fluorescence image split in green channel (top panel) and red channel (bottom panel). The significantly high non-flat background was removed by the rolling ball algorithm. (b) Resulting images exhibit a flat background, from which the fluorescence spots are well distinguishable. (c) Intensity profile evaluated along the yellow and blue lines highlighted in panels (a) and (b), respectively. (d) Example of processed fluorescence image in which the green and red channels are merged again. Corresponding intensity histograms obtained for (e) green channel and (f) red channel. 


\section{Section S6. Analysis of the bright spots.}

In order to demonstrate the assumption that the fluorescence of each bright spot corresponds to the light emitted by one single fluorophore, we firstly considered the lowest PfLDH concentrations ( $1 \mathrm{fM}$ ) we investigated (Figure $3 \mathrm{~b}$ in the main text). The corresponding number of analytes in $1 \mathrm{~mL}$ volume (i.e., the volume we used throughout the work) is $6 \times 10^{5}$. Thus, in principle we can have $6 \times 10^{5} P f L D H$ proteins over the whole substrate $\left(10 \times 8 \mathrm{~mm}^{2}\right)$ and, hence, $2 \times 10^{4}$ analytes can be virtually housed in the microscope field of view (FOM) $(1.66 \times$ $1.40 \mathrm{~mm}^{2}$ ). In ideal conditions, $2 \times 10^{4}$ fluorophores bind the analytes in the FOM. The photodetector contains $5.5 \times 10^{6}$ pixels (see Section S5).

We can now evaluate the probability that two fluorophores lie on an area of the substrate whose fluorescence would be collected by one pixel $\left(0.650 \times 0.650 \mu \mathrm{m}^{2}\right.$, see Section S5). Since the number of fluorophores $\left(2 \times 10^{4}\right)$ is much smaller than the number of pixels $\left(5.5 \times 10^{6}\right)$, we can adopt the Poisson statistics to retrieve the probability $P(k)$ that $k$ fluorophores are placed in one pixel ${ }^{4}$

$$
P(k)=\mathrm{e}^{-\frac{m}{n}} \frac{\left(\frac{m}{n}\right)^{k}}{k !}
$$

where $m$ is the number of fluorophores and $n$ is the number of pixels. It results $P(2)=$ $0.0005 \%$. Therefore, we can safely assert that bright spots appearing in fluorescence images at very low $P f \mathrm{LDH}$ concentration (femtomolar level) arise from individual fluorophores.

Moving to the next PfLDH concentrations we explored $1 \mathrm{pM}, 1 \mathrm{nM}$, and $1 \mu \mathrm{M}$, the number of fluorophores housed in the FOM in ideal conditions is $2 \times 10^{7}, 2 \times 10^{10}$, and $2 \times 10^{13}$, respectively. Since $m>n$, we evaluated the probabilities $P(0)$ that one pixel is vacant. Since $P(0)=\mathrm{e}^{-m / n}$, it results $43 \%$ at $1 \mathrm{pM}$ and negligible values at $1 \mathrm{nM}$ and $1 \mu \mathrm{M}$. However, considering $l$ dark pixels, the probabilities are much lower $P^{(l)}(0)=\mathrm{e}^{-m l / n}$. For instance, $P^{(10)}(0)=0.02 \%$ at $1 \mathrm{pM}$. Nevertheless, in real measurements a much larger number of dark pixels were present as compared to the number of bright spots even at the largest PfLDH concentration (Figure $3 b$ in the main text). This result can be reasonably achieved only if $m \ll$ $n$.

Such a discrepancy can be justified considering a low system efficiency that depends on (i) the binding affinities between Abs-Au, Abs-PfLDH and Apts*-PfLDH and (ii) the fluidic efficiency in each step. Thus, only a small amount of the available fluorophores would be housed on the substrate and only a tiny fraction of them would experience a significant FE. Therefore, we can assume that the condition $m<n$ is hold even at large concentrations (up 
to micromolar level) and, hence, the probability $P(l)$ that $l \geq 2$ fluorophores lie on one pixel is low.

The previous argumentation is coherent with the linearity exhibited by the correlation curves fluorescence versus spot area (Figure S6). The slope of the linear regressions is independent from the PfLDH concentration revealing that the mean fluorescence intensity per pixel at low analyte concentrations (femtomolar level) is hold even at high concentrations (micromolar level). In addition, the number of bright spots whose intensity/area ratio lies outside the linear regression is relatively low even if it enlarges as the analyte concentration increases. In particular, these spots arose from nearby fluorophores whose light was collected by the same pixels of the photodetector so to increase the spot brightness while holding the spot size. In conclusion, we can safely assert that most of the observed bright spots arise from individual fluorophores.

Finally, we can retrieve the ratio between the measured FE (of approximately 28) by considering the ratio between the slopes of the corresponding correlation curves (fluorescence vs spot area)

$$
\frac{\left\langle F E_{\mathrm{Cy} 5}^{\mathrm{obs}}\right\rangle}{\left\langle F E_{5 \mathrm{FAM}}^{\mathrm{obs}}\right\rangle} \sim \frac{m_{\mathrm{Cy} 5}}{Q Y_{\mathrm{Cy} 5}^{0}} \frac{Q Y_{5 \mathrm{FAM}}^{0}}{m_{5 \mathrm{FAM}}}
$$

where $Q Y_{5 \mathrm{FAM}}^{0}=0.83^{5}$ and $Q Y_{\mathrm{Cy} 5}^{0}=0.27 .{ }^{6}$ Thus, we obtain a value of $\sim 20$ by Equation (2) that is consistent with that evaluated by considering the measured FE. 
(a)

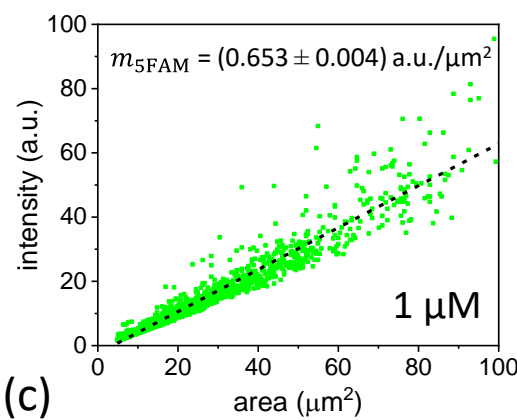

(c)

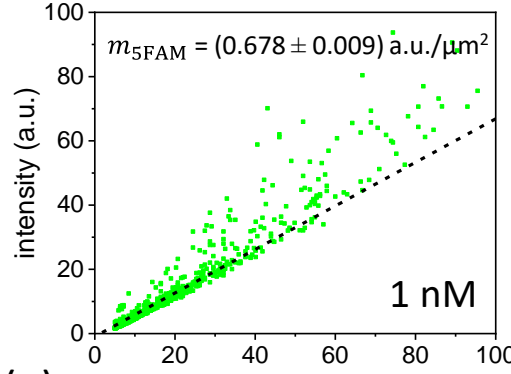

(e)

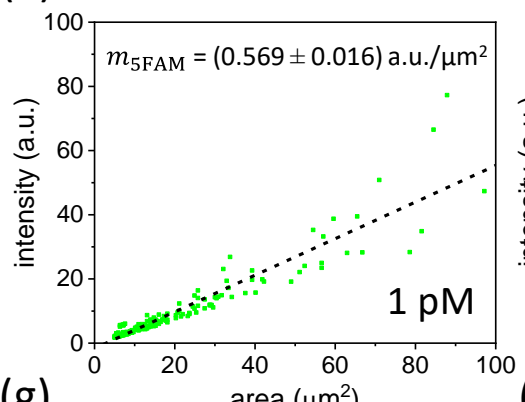

(g)

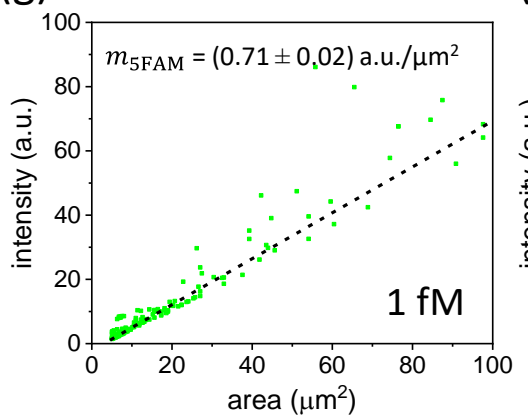

(b)
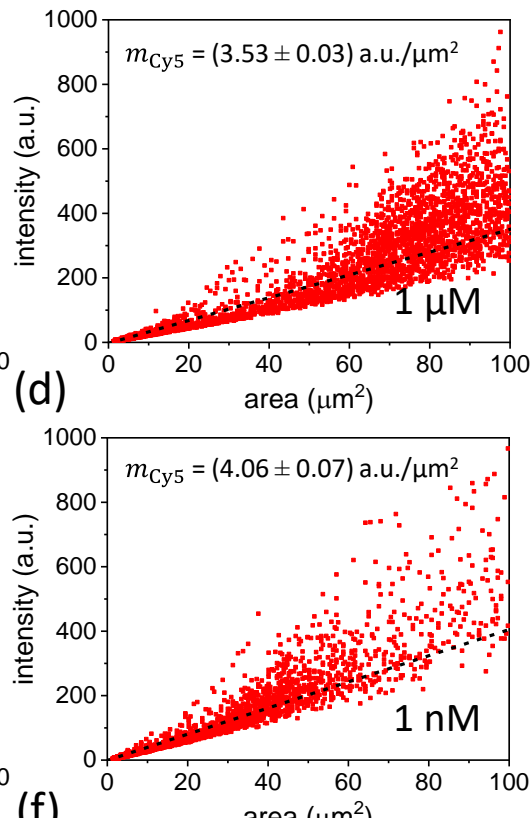

(f)
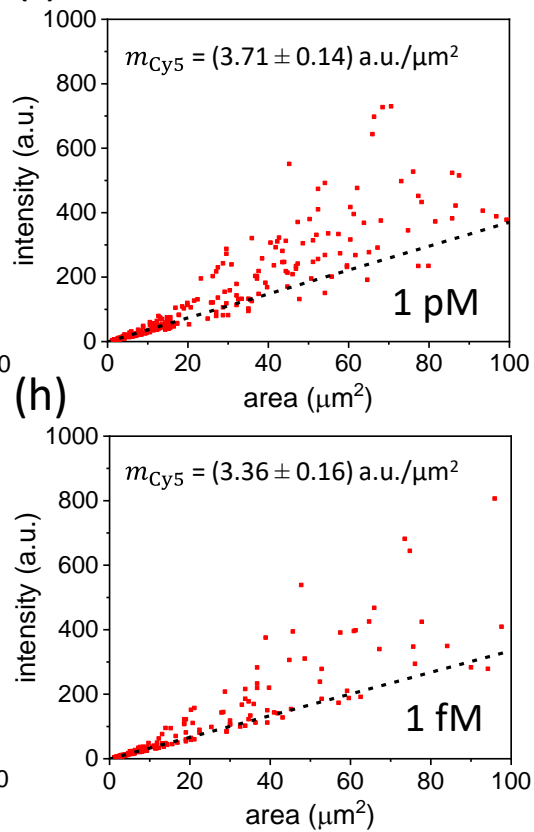

Figure S6. Correlation intensity vs spot area explored by (a,c,e,g) 5-FAM dye and (b,d,f,h) Cy5 dye at $P f \mathrm{LDH}$ concentrations of (a,b) $1 \mu \mathrm{M},(\mathrm{c}, \mathrm{d}) 1 \mathrm{nM},(\mathrm{e}, \mathrm{f}) 1 \mathrm{pM}$ and $(\mathrm{g}, \mathrm{h}) 1 \mathrm{fM}$ spiked in human whole blood (Pearson's $r=0.95$ ). 
Section S7. Off-resonance simulations.

(a)

(b)

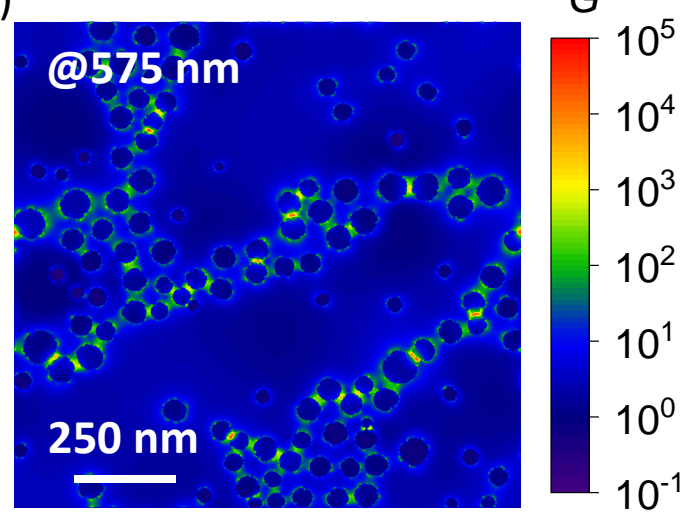

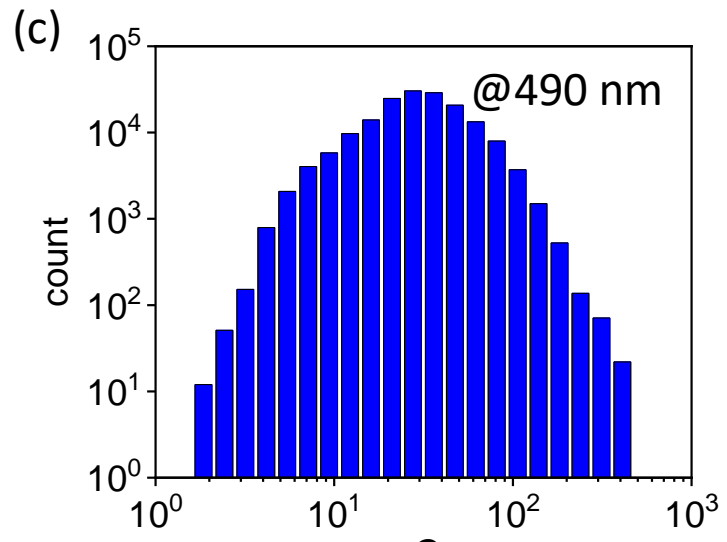

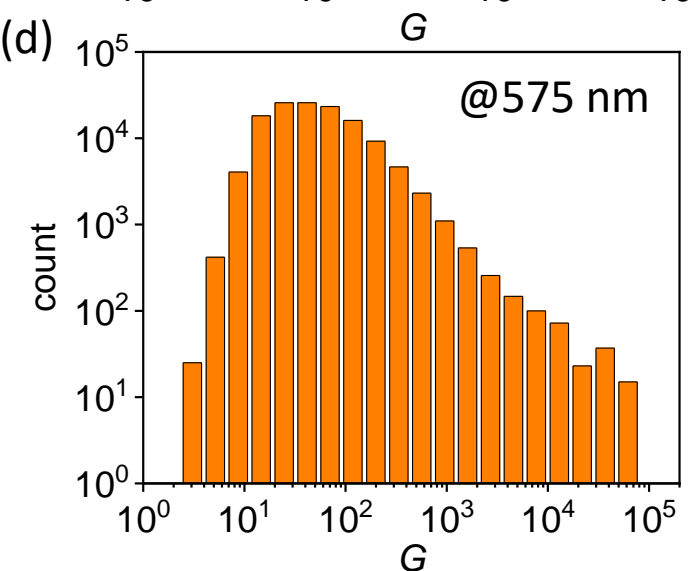

Figure S7. Simulated electric field intensity of the substrate worked out at wavelength (a) $490 \mathrm{~nm}$ and (b) 575 Histograms of the $G$ value distribution evaluated in annulus-shaped regions around nanoparticles (5 nm thickness, $5 \mathrm{~nm}$ offset from particle surface) at wavelength (c) $490 \mathrm{~nm}$ and (d) $575 \mathrm{~nm}$. 


\section{Section S8. Analysis of the electric field.}

The electric field $E_{n}$ around the $n$-th nanoparticle was evaluated in the $x$ - $y$ plane in annuluses of $5 \mathrm{~nm}$ thickness and $5 \mathrm{~nm}$ offset from the nanoparticle surface by Equation (3)

$$
E_{n}(r, \varphi)=E\left(x_{n}+r \cos \varphi, y_{n}+r \sin \varphi\right)
$$

where $r$ is the distance from the nanoparticle centroid ( $1 \mathrm{~nm}$ spatial resolution), $\varphi$ is the azimuthal angle $\left(1^{\circ}\right.$ angular resolution), and $\left(x_{n}, y_{n}\right)$ are the centroid coordinates. Note that $r$ values range from $D_{n} / 2+5 \mathrm{~nm}$ to $D_{n} / 2+10 \mathrm{~nm}$, in which $D_{n}$ is the diameter of the $n$-th nanoparticle measured by SEM micrographs (see Section S1). We implemented Equation (3) in a Python code that excluded multiple counting in overlapping annuluses. Figure S8 shows two representative magnifications of the electric field intensity worked out with perfectly spherical gold nanoparticles and rough gold nanoparticles, in which the shaded red annuluses highlight the only regions where the $G$ values were evaluated.

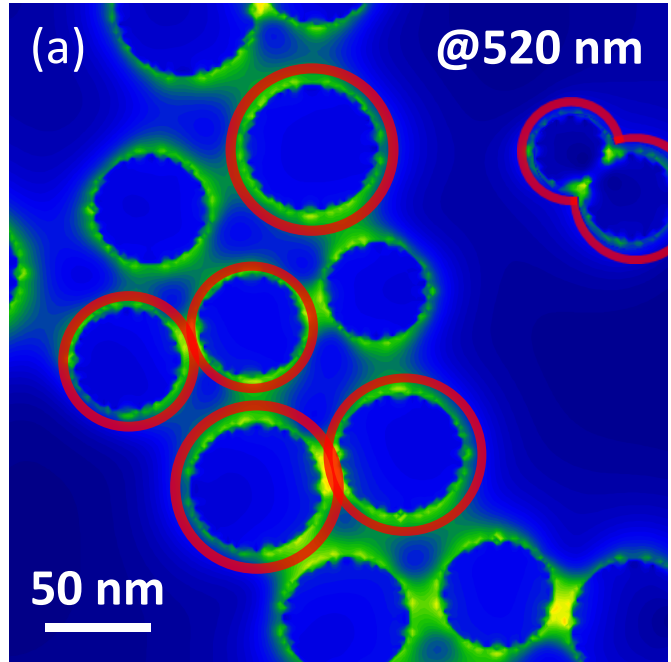

G $\begin{array}{lllll}10^{3} & 10^{2} & 10^{1} & 10^{0} & 10^{-1}\end{array}$

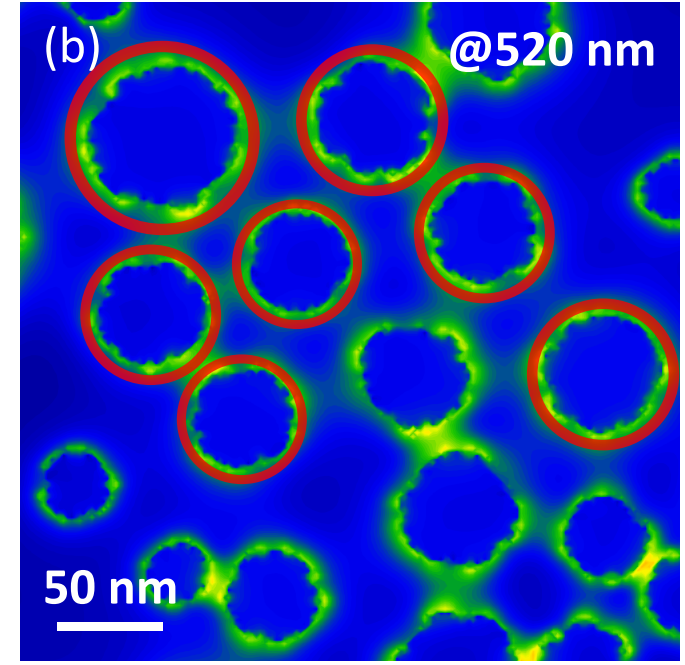

G $10^{4} 10^{3} 10^{2} 10^{1} 10^{0} 10^{-1}$

Figure S8. Electric field intensity distributions of (a) perfectly spherical gold nanoparticles and (b) rough gold nanoparticles worked out at $520 \mathrm{~nm}$ wavelength. The shaded red annuluses $(5 \mathrm{~nm}$ thickness, $5 \mathrm{~nm}$ offset from the nanoparticle surface) highlight the regions where the $G$ values were evaluated. Note that in the case of rough nanoparticles, the annulus offset is determined by considering an ideal spherical surface embedding the nanoparticle roughness. 
Section S9. Electromagnetic response of the substrate with perfectly spherical nanoparticles.
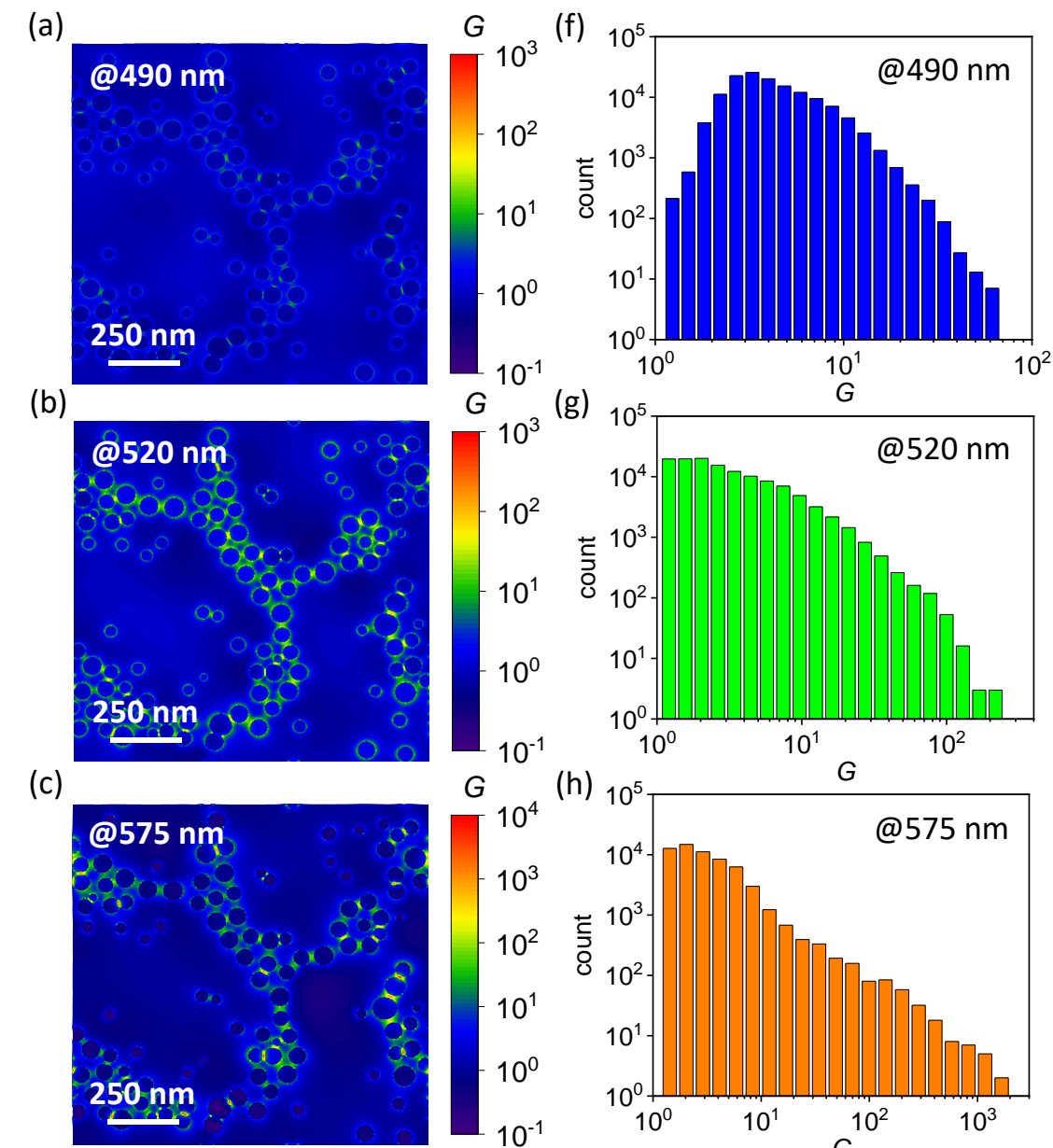

(d)
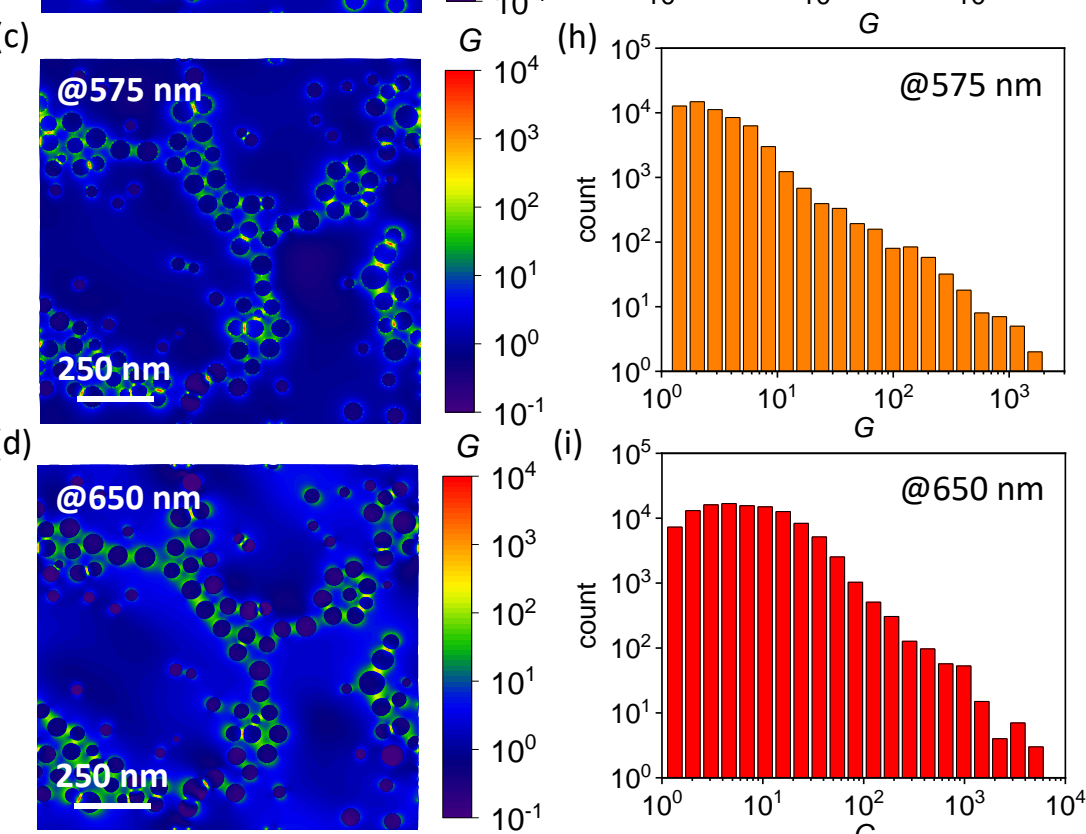

(e)
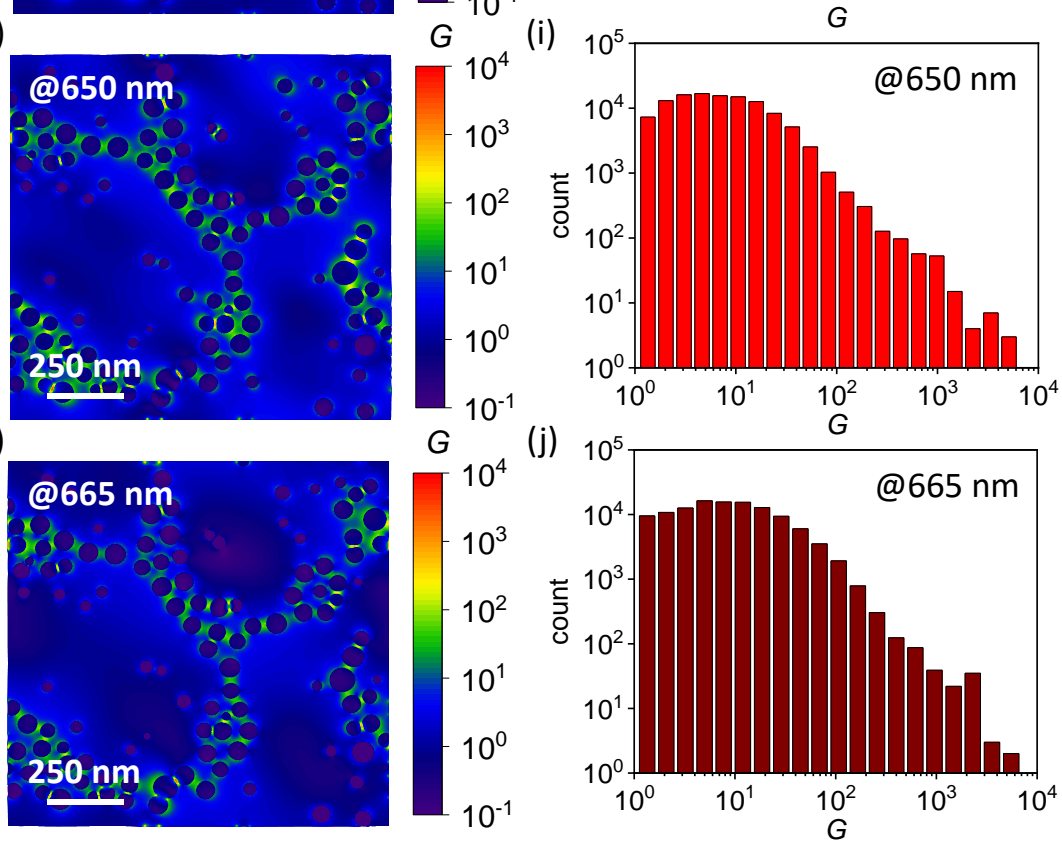

Figure S9. Simulated electric field intensity of the substrate in the case of perfectly spherical AuNPs worked out at wavelength (a) $490 \mathrm{~nm}$, (b) $520 \mathrm{~nm}$, (c) $575 \mathrm{~nm}$, (d) $650 \mathrm{~nm}$, and (e) $665 \mathrm{~nm}$. Histograms of the $G$ value distribution evaluated in annulus-shaped regions around nanoparticles ( $5 \mathrm{~nm}$ thickness, $5 \mathrm{~nm}$ offset from particle surface) at wavelength (f) $490 \mathrm{~nm},(\mathrm{~g})$ $520 \mathrm{~nm}$, (h) $575 \mathrm{~nm}$, (i) $650 \mathrm{~nm}$, and (j) $665 \mathrm{~nm}$. 
Section S10. Electromagnetic response of non-spherical nanoparticles.

(a)

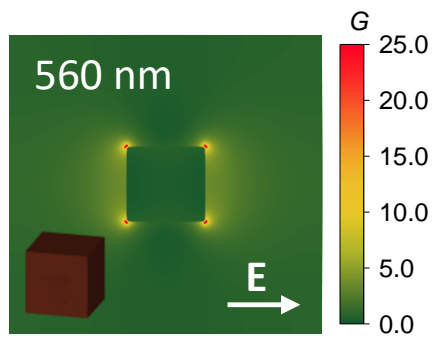

(b)

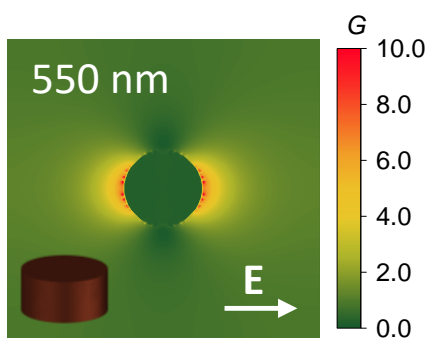

(c)

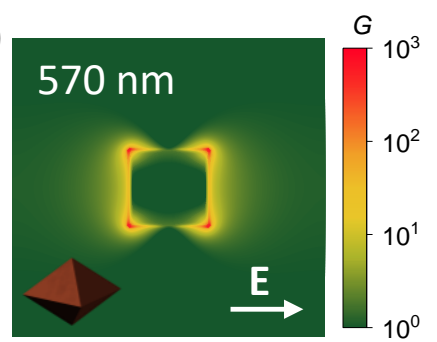

(d)

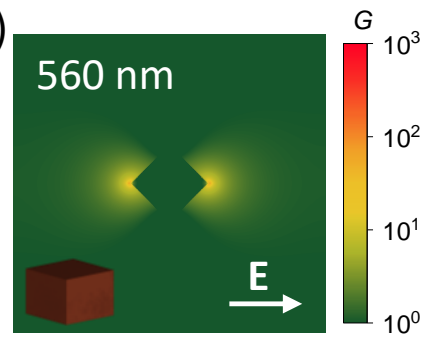

(e)

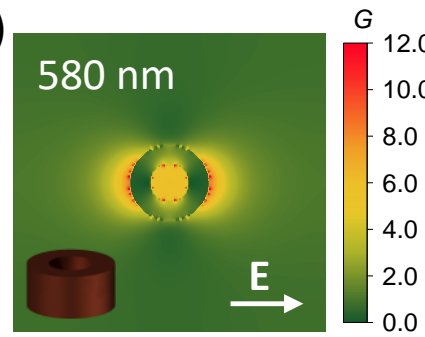

(f)

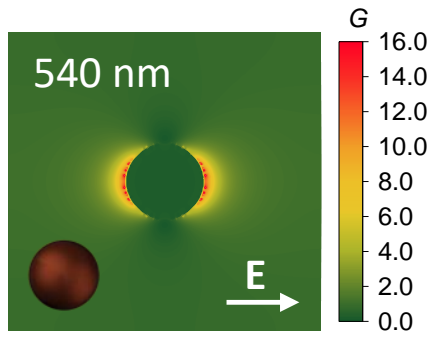

(g)

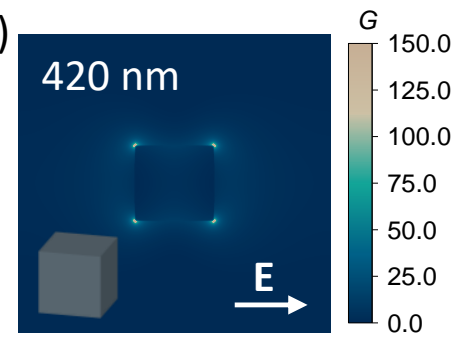

(h)

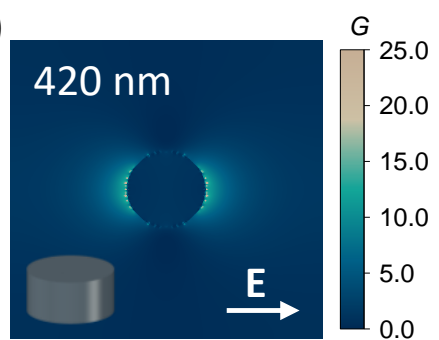

(i)

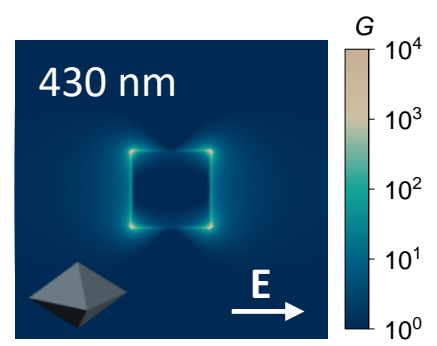

(j)

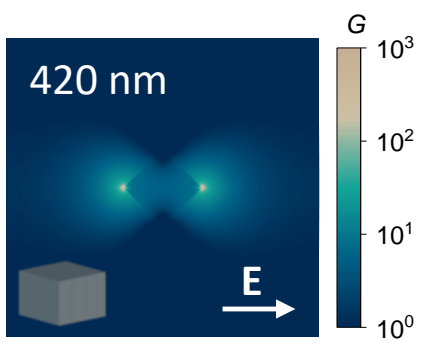

(k)

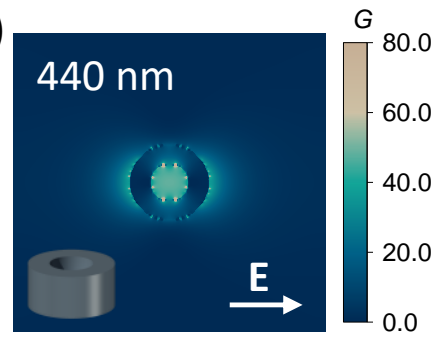

(I)

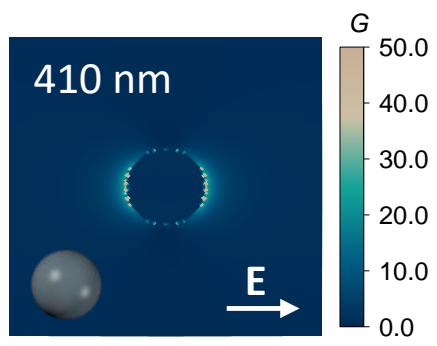

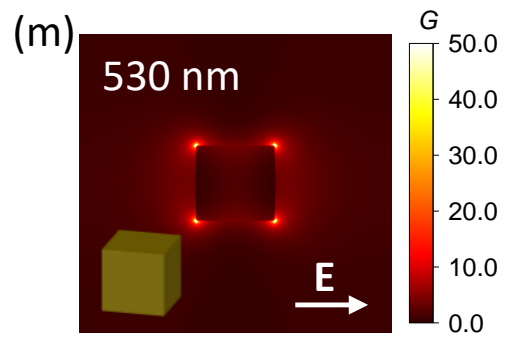

(n)

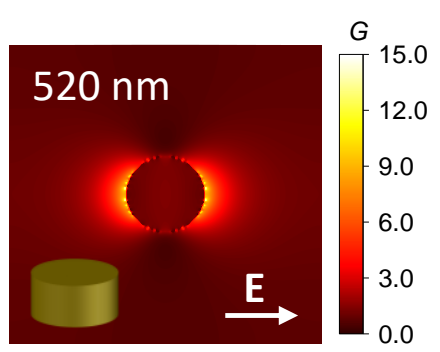

(o)

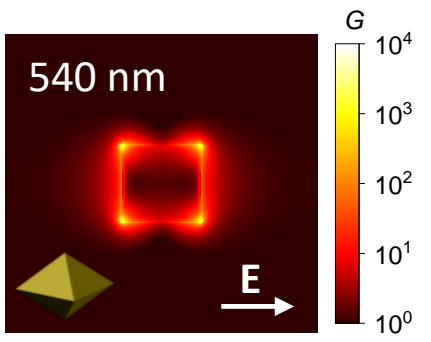

(p)

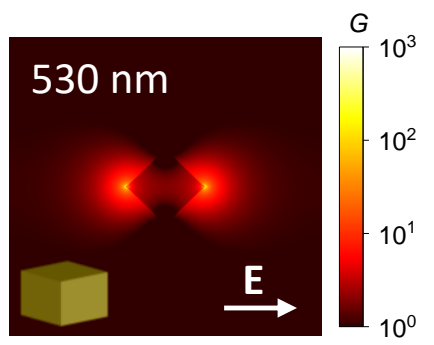

(q)

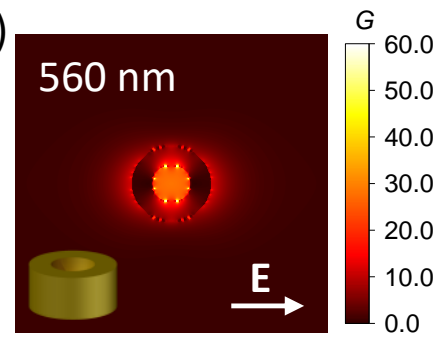

(r)

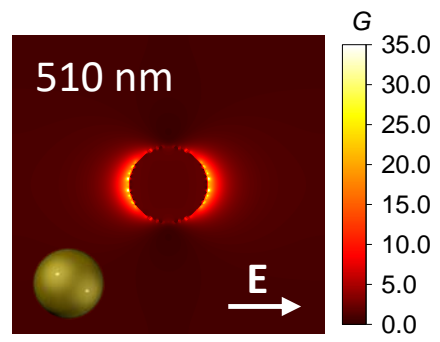

Figure S10.A. Simulated electric field intensity of a copper (left panels), silver (middle panels), gold (right panels) (a,g,m) cube, (b,h,n) disk, (c,i,o) octahedron, (d,j,p) rhombus, (e,k,q) ring, and (f,l,r) sphere worked out at the corresponding resonance wavelength. 
(a)

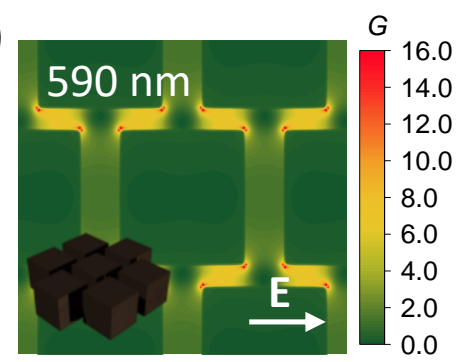

(b)

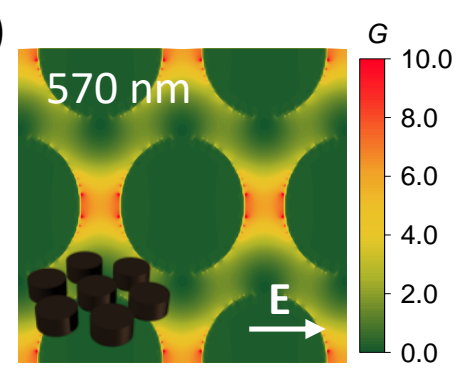

(c)

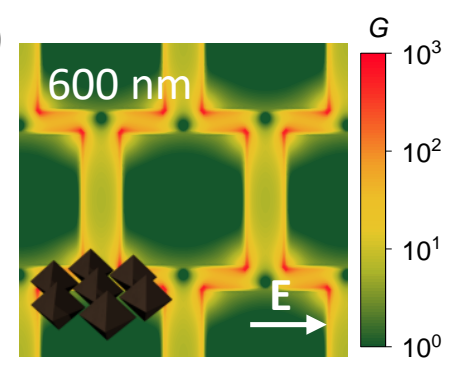

(d)

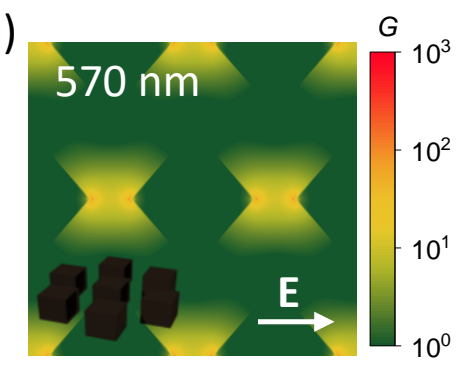

(e)

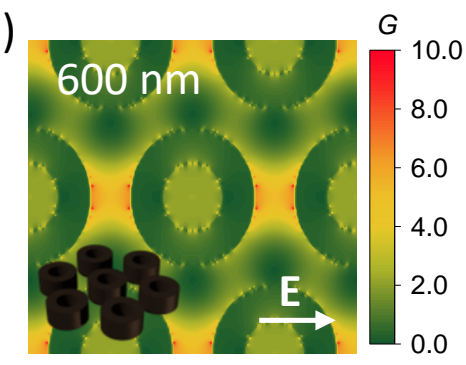

(f)

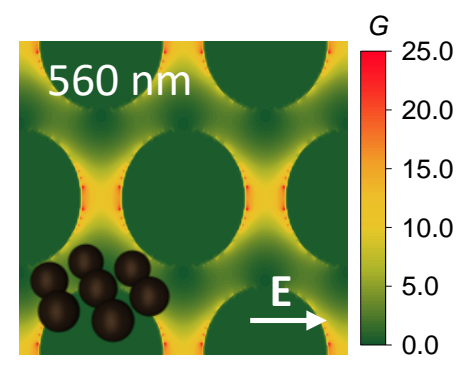

(g)

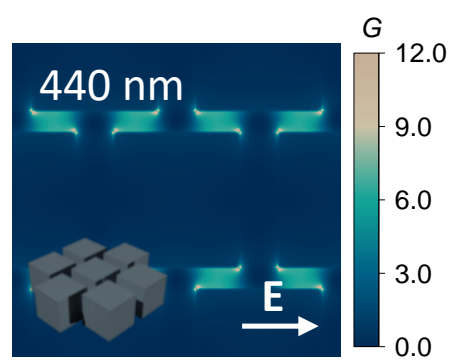

(h)

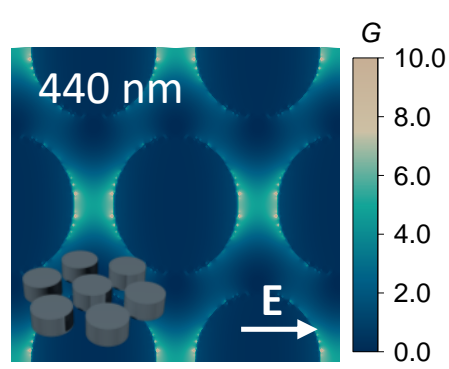

(i)

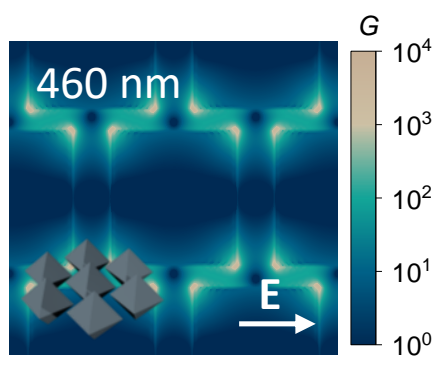

(j)

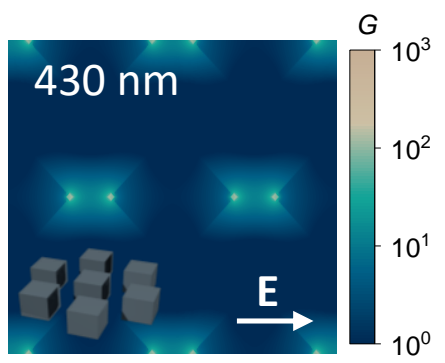

(k)

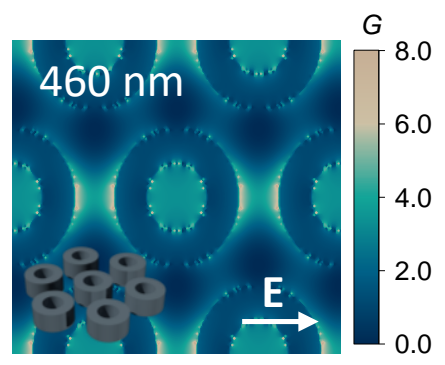

(I)

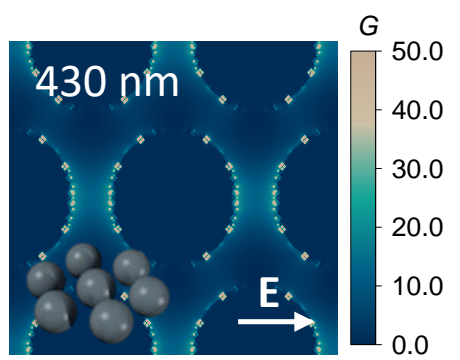

(m)

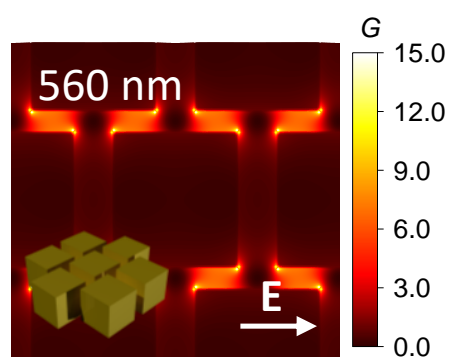

(n)

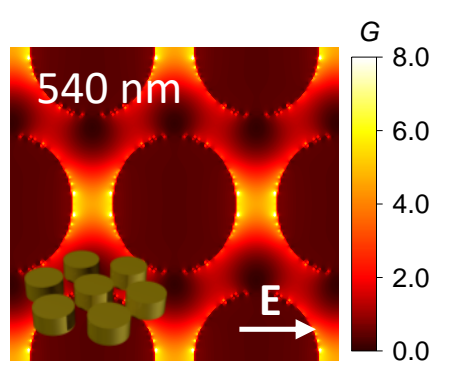

(o)

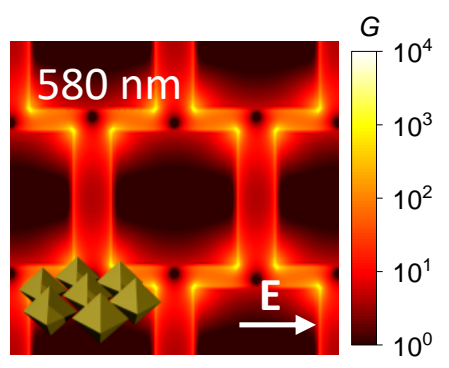

(p)

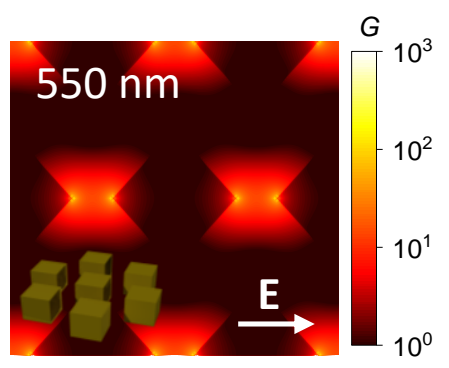

(q)

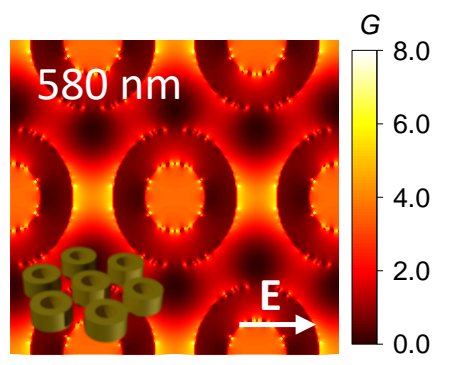

(r)

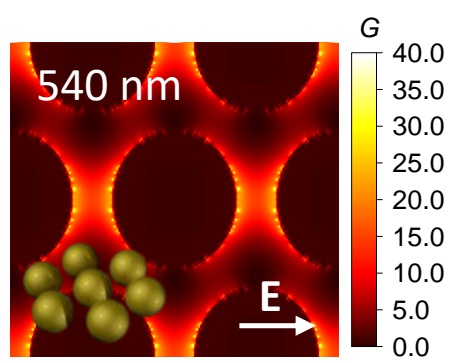

Figure S10.B. Simulated electric field intensity of a hexagonal lattice of copper (left panels), silver (middle panels), gold (right panels) (a,g,m) cubes, (b,h,n) disks, (c,i,o) octahedra, (d,j,p) rhombi, (e,k,q) rings, and $(\mathrm{f}, 1, \mathrm{r})$ spheres worked out at the corresponding resonance wavelength. 


\section{Section S11. Fluorescence intensity in non-PEF conditions.}

To measure the intensity $\left\langle I_{0}\right\rangle$, a volume of $100 \mu \mathrm{L}$ containing $250 \mathrm{fmol}$ of Apts* (approximately 1.5 $\times 10^{11}$ fluorophores) was drop-casted onto a microscope slide. After drying, the 5-FAM (Cy5) fluorophores resulted to be confined within a circular region of $12 \mathrm{~mm}(13 \mathrm{~mm})$ diameter surrounded by an annulus (i.e., coffee ring) of $0.15 \mathrm{~mm}(0.10 \mathrm{~mm})$ thickness whose intensity was 10 -fold (4-fold) higher than that measured in the inner region (Figure S11).

The whole fluorescence intensity $F_{0}$ of the dried drop was measured by sampling the drop area in 100 regions of size $350 \times 350 \mu \mathrm{m}^{2}$ in the inner region and $70 \times 70 \mu \mathrm{m}^{2}$ on the annulus. Then, the resulting averages were scaled to the whole inner region $\left(\sim 113 \mathrm{~mm}^{2}\right.$ for 5-FAM dye and $\sim 133 \mathrm{~mm}^{2}$ for Cy5 dye) and coffee ring area $\left(\sim 5.7 \mathrm{~mm}^{2}\right.$ for 5-FAM dye and $\sim 4.1 \mathrm{~mm}^{2}$ for Cy5 dye). Thus, the mean intensity $\left\langle I_{0}\right\rangle$ provided by individual fluorophores in free-space condition is

$$
\left\langle I_{0}\right\rangle=\frac{F_{0}}{N_{0}}
$$

where $N_{0}$ the number of fluorophores contained in the drop.
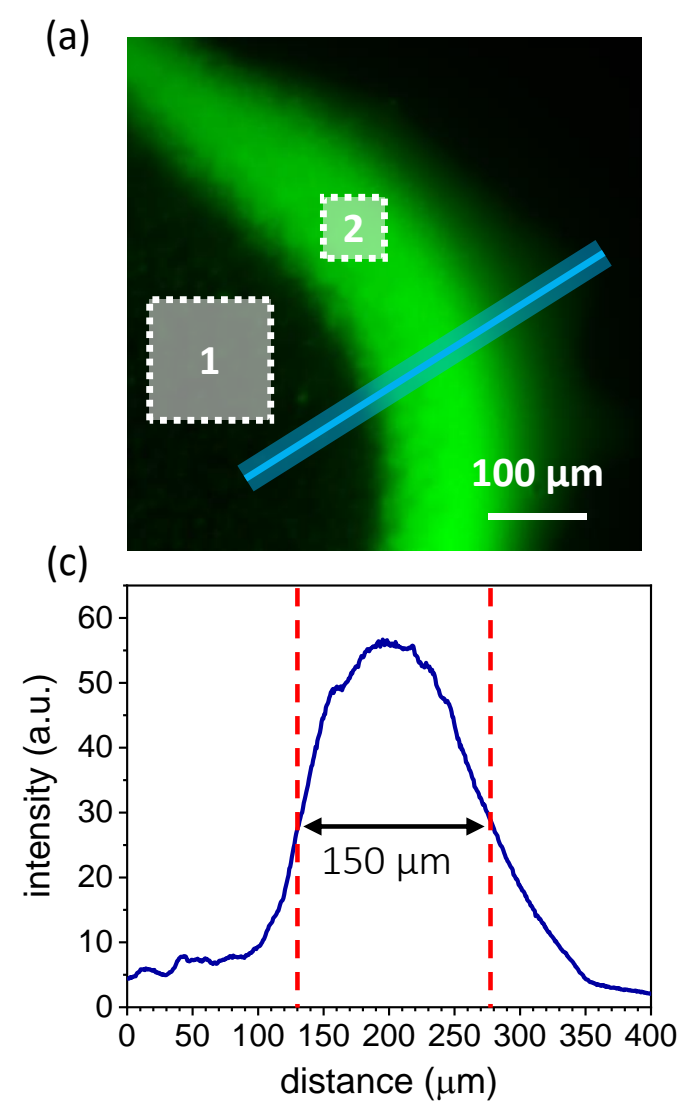

(b)

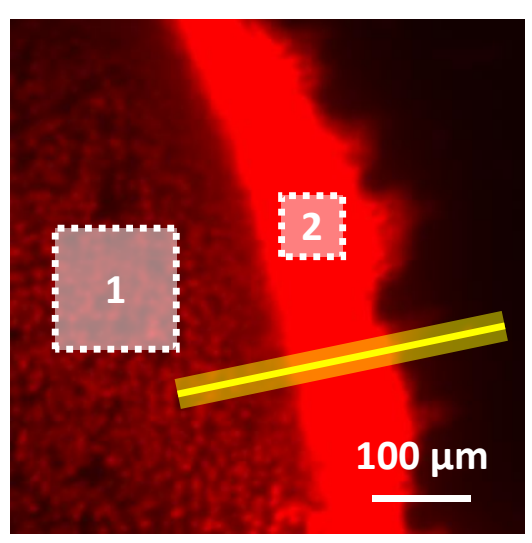

(d)

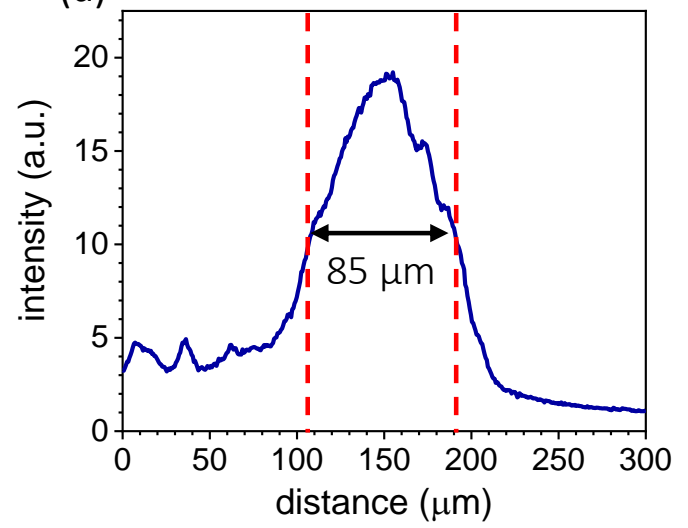

Figure S11. Fluorescence image of (a) 5-FAM-Apts* and (b) Cy5-Apts* dried drops. The drop was sampled in 100 regions of size $350 \times 350 \mu \mathrm{m}^{2}$ in the inner area (white squares 1 , not in scale) and 70 $\times 70 \mu \mathrm{m}^{2}$ on the annulus (white squares 2). (c,d) Ring thickness measured as FWHM of the intensity profile evaluated along the light blue and yellow lines highlighted in panels (a) and (b), respectively. Adapted under the terms of the CC-BY license. ${ }^{7,8}$ Copyright 2020 and 2021, The Authors, published by Springer Nature. 


\section{Section S12. Chemicals and materials.}

Gold(III) chloride trihydrate $\left(\mathrm{HAuCl}_{4} \cdot 3 \mathrm{H}_{2} \mathrm{O}\right)$, silver nitrate $\left(\mathrm{AgNO}_{3}\right)$, ascorbic acid and toluene $(99.8 \%)$ were purchased from Sigma-Aldrich; ethanol ( $\geq 99.5 \%)$, 2-propanol $(\geq$ 99.5\%) and acetone $(\geq 99.0 \%)$ were purchased from Merck Millipore; hexadecyltrimethylammonium bromide (CTAB) $(\geq 99.0 \%$ ) was purchased from Fluka; bovine serum albumin (BSA) (fraction V IgG free, fatty acid poor) was purchased from Gibco. Diblock copolymers (P3807-S2VP) were purchased from Polymer Source Inc. (Dorval, Canada) and were made by polystyrene (x)-b-2-poly-vinylpyridine $(y)(\operatorname{PS}(x)-b-\operatorname{P} 2 \mathrm{VP}(y))$, in which $x=325000 \mathrm{~g} / \mathrm{mol}$ and $y=92000 \mathrm{~g} / \mathrm{mol}$ are the molecular weight of polystyrene (PS) and poly(2-vinylpyridine) (P2VP), respectively. Ultrapure deionized water used for all aqueous solutions was dispensed by Milli-Q® ${ }^{\circledR}$ system (18.2 $\mathrm{M} \Omega \mathrm{cm}$ resistivity). $10 \mathrm{mM}$ phosphate-buffered saline (PBS) ( $\mathrm{NaCl} 10 \mathrm{mM}, \mathrm{NaH}_{2} \mathrm{PO}_{4} 10 \mathrm{mM}, \mathrm{Na}_{2} \mathrm{HPO}_{4} 10 \mathrm{mM}, \mathrm{MgCl}_{2}$ $1 \mathrm{mM}, \mathrm{pH} 7.1)$ and $25 \mathrm{mM}$ Tris- $\mathrm{HCl}$ buffer ( $\mathrm{NaCl} 100 \mathrm{mM}$, imidazole $20 \mathrm{mM}$, Tris $25 \mathrm{mM}$, $\mathrm{HCl} 25 \mathrm{mM}, \mathrm{pH}$ 7.5) were prepared by dissolving the reagents (purchased from SigmaAldrich) in ultrapure water. Pan malaria antibody (monoclonal anti-PLDH antibody clone 19g7) was produced by Vista Laboratory Services (Langley, USA). The recombinant PfLDH and $P v \mathrm{LDH}$ were obtained from bacterial expression as previously described. ${ }^{9}$ The malaria 2008s aptamer labelled with 5-FAM (analogously, Cy5) tag (5'-5-FAM(Cy5)-CTG GGC GGT AGA ACC ATA GTG ACC CAG CCG TCT AC-3') was synthesized by Friz Biochem GmbH (Neuried, Germany). Millex ${ }^{\circledR}$ syringe filters (pore size $0.20 \mu \mathrm{m}$ ) with hydrophilic polytetrafluoroethylene membrane were purchased from Merck Millipore; Superslip ${ }^{\mathrm{TM}}$ cover slips (borosilicate glass, thickness $0.13-0.17 \mathrm{~mm}$ ) were purchased from Thermo Fisher Scientific. 


\section{Section S13. Photochemical immobilization technique.}

The PIT consists of UV irradiation of Abs that produces the selective photoreduction of the disulfide bridge in specific cysteine-cysteine/tryptophan (Cys-Cys/Trp) triads, ${ }^{10}$ which are a typical structural feature of the immunoglobulin G (IgG). ${ }^{11}$ The UV excitation of the $\operatorname{Trp}$ residue leads to the generation of solvated electrons that are captured by the nearby disulphide bridge resulting in its destabilization and subsequent breakage of the Cys-Cys bond. The breakage of such Cys-Cys bonds in both $\mathrm{Ab}$ Fab fragments produces four free thiol groups (Figure S12a), two of which are able to interact with the proximal gold surface giving rise to a covalent $\mathrm{Ab}$ tether. Functionalization by PIT assures both close-packing and control over the orientation of the immobilized Abs, with one of their binding sites exposed to the surrounding environment (Figure S12b).

The UV source (Trylight, Promete S.r.1.) consisted of two U-shaped low-pressure mercury lamps (6 W at $254 \mathrm{~nm}$ ) in which a standard $10 \mathrm{~mm}$ quartz cuvette could be easily housed (Figure S12c). By considering the wrapping geometry of the lamps and the proximity of the cuvette, the irradiation intensity used for the thiol group production was approximately 0.3 $\mathrm{W} / \mathrm{cm}^{2}$. Such an intensity is low enough to avoid any significant photolysis of the disulfide bridge that poorly absorbs at $254 \mathrm{~nm} .^{12}$

(a)

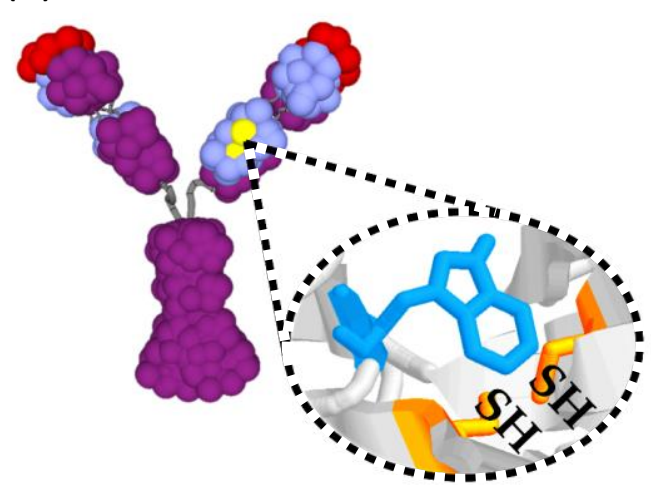

(b)

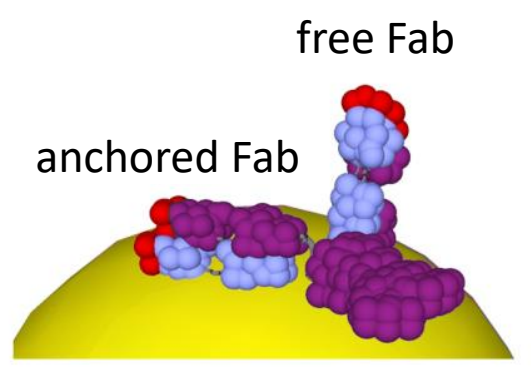

(c)

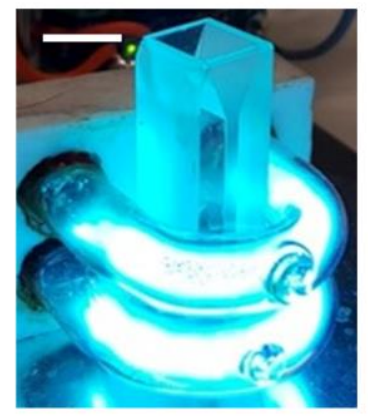

Figure S12. (a) UV irradiation of Abs leads to the selective production of four thiol groups (two of them are not visible in the figure). (b) The opposite position of the thiols with respect to the plane containing the Fabs allows the Abs to be immobilized with one of their binding sites exposed to the surrounding environment. (c) Low pressure mercury U-shaped UV lamps used to carry out the thiol activation in antibody Fabs. The length of the scale bar in the top-left corner is $1 \mathrm{~cm}$. Adapted under the terms of the CC-BY license. ${ }^{8}$ Copyright 2021, The Authors, published by Springer Nature. 
Section S14. Microfluidic system.

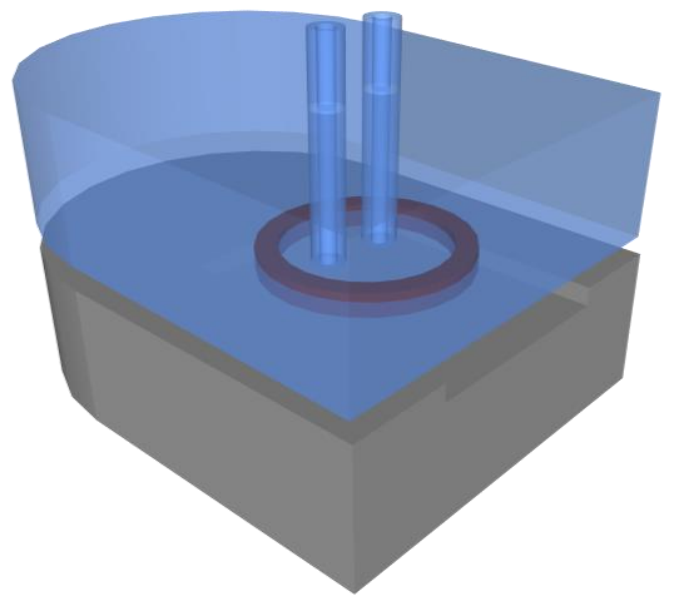

Figure S13. Sketch of the fluidic cell used to promote an effective interaction of the irradiated Abs contained into the aqueous solution with the nanostructured substrate. Adapted under the terms of the CC-BY license. ${ }^{8}$ Copyright 2021, The Authors, published by Springer Nature. 
Section S15. Preparation of buffer solutions.

Table S2. Tris buffer solution was prepared by dissolving the reagents into $500 \mathrm{~mL}$ of ultrapure water under vigorous stirring at room temperature. The buffer $\mathrm{pH}$ was adjusted to 7.5 by adding $750 \mu \mathrm{L}$ of $\mathrm{HCl} 37 \%$.

\begin{tabular}{|c|c|c|c|}
\hline Chemical & State & Added amount & Final concentration \\
\hline $\mathrm{NaCl}$ & powder & $2.922 \mathrm{~g}$ & $100 \mathrm{mM}$ \\
\hline Imidazole & powder & $0.681 \mathrm{~g}$ & $20 \mathrm{mM}$ \\
\hline Tris base & powder & $1.515 \mathrm{~g}$ & $25 \mathrm{mM}$ \\
\hline $\mathrm{HCl} 37 \%$ & liquid & $397 \mu \mathrm{L}$ & - \\
\hline
\end{tabular}

Table S3. PBS solution was prepared by dissolving the reagents into $100 \mathrm{~mL}$ of ultrapure water under vigorous stirring at room temperature. The buffer $\mathrm{pH}$ was adjusted to 7.1 by adding $250 \mu \mathrm{L}$ of $\mathrm{NaOH}$ (4 M).

\begin{tabular}{|c|c|c|c|}
\hline Chemical & State & Added amount & Final concentration \\
\hline $\mathrm{NaCl}$ & powder & $585 \mathrm{mg}$ & $100 \mathrm{mM}$ \\
\hline $\mathrm{NaH}_{2} \mathrm{PO}_{4}$ & powder & $140 \mathrm{mg}$ & $10 \mathrm{mM}$ \\
\hline $\mathrm{Na}_{2} \mathrm{HPO}_{4}$ & powder & $140 \mathrm{mg}$ & $10 \mathrm{mM}$ \\
\hline $\mathrm{MgCl}_{2}$ & powder & $9.5 \mathrm{mg}$ & $1 \mathrm{mM}$ \\
\hline
\end{tabular}




\section{References}

(1) Haynes, W. M. CRC Handbook of Chemistry and Physics; Haynes, W. M., Lide, D. R., Bruno, T. J., Eds.; CRC Press, 2016. https://doi.org/10.1201/9781315380476.

(2) Palik, E. D. Handbook of Optical Constants of Solids; Elsevier, 1985. https://doi.org/10.1016/C2009-0-20920-2.

(3) Bérenger, J. P. Perfectly Matched Layer (PML) for Computational Electromagnetics. Synth. Lect. Comput. Electromagn. 2007, 8 (1), 1-120. https://doi.org/10.2200/S00030ED1V01Y200605CEM008.

(4) Raab, M.; Steger, A. "Balls into Bins" — a Simple and Tight Analysis. In Lecture Notes in Computer Science (including subseries Lecture Notes in Artificial Intelligence and Lecture Notes in Bioinformatics); Luby, M., Rolim, J. D. P., Serna, M., Eds.; Springer Berlin Heidelberg: Berlin, Heidelberg, 1998; Vol. 1518, pp 159-170. https://doi.org/10.1007/3-54049543-6_13.

(5) Povedailo, V. A.; Lysenko, I. L.; Tikhomirov, S. A.; Yakovlev, D. L.; Tsybulsky, D. A.; Kruhlik, A. S.; Fan, F.; Martynenko-Makaev, Y. V.; Sharko, O. L.; Duong, P. V.; et al. Fluorescent Properties of Carboxyfluorescein Bifluorophores. J. Fluoresc. 2020, 30 (3), 629635. https://doi.org/10.1007/s10895-020-02535-w.

(6) Mujumdar, R. B.; Ernst, L. A.; Mujumdar, S. R.; Lewis, C. J.; Waggoner, A. S. Cyanine Dye Labeling Reagents: Sulfoindocyanine Succinimidyl Esters. Bioconjug. Chem. 1993, 4 (2), 105-111. https://doi.org/10.1021/bc00020a001.

(7) Minopoli, A.; Della Ventura, B.; Lenyk, B.; Gentile, F.; Tanner, J. A.; Offenhäusser, A.; Mayer, D.; Velotta, R. Ultrasensitive Antibody-Aptamer Plasmonic Biosensor for Malaria Biomarker Detection in Whole Blood. Nat. Commun. 2020, 11 (1), 6134. https://doi.org/10.1038/s41467-020-19755-0.

(8) Minopoli, A.; Della Ventura, B.; Campanile, R.; Tanner, J. A.; Offenhäusser, A.; Mayer, D.; Velotta, R. Randomly Positioned Gold Nanoparticles as Fluorescence Enhancers in AptaImmunosensor for Malaria Test. Microchim. Acta 2021, 188 (3), 88. https://doi.org/10.1007/s00604-021-04746-9.

(9) Cheung, Y.-W.; Dirkzwager, R. M.; Wong, W.-C.; Cardoso, J.; D’Arc Neves Costa, J.; Tanner, J. A. Aptamer-Mediated Plasmodium-Specific Diagnosis of Malaria. Biochimie 2018, 145, 131-136. https://doi.org/10.1016/j.biochi.2017.10.017.

(10) Neves-Petersen, M. T.; Gryczynski, Z.; Lakowicz, J.; Fojan, P.; Pedersen, S.; Petersen, E.; Bjørn Petersen, S. High Probability of Disrupting a Disulphide Bridge Mediated by an Endogenous Excited Tryptophan Residue. Protein Sci. 2002, 11 (3), 588-600. 
https://doi.org/10.1110/ps.06002.

(11) Ioerger, T. R.; Du, C.; Linthicum, D. S. Conservation of Cys-Cys Trp Structural Triads and Their Geometry in the Protein Domains of Immunoglobulin Superfamily Members. Mol. Immunol. 1999, 36 (6), 373-386. https://doi.org/10.1016/S0161-5890(99)00032-2.

(12) Neves-Petersen, M. T.; Klitgaard, S.; Pascher, T.; Skovsen, E.; Polivka, T.; Yartsev, A.; Sundström, V.; Petersen, S. B. Flash Photolysis of Cutinase: Identification and Decay Kinetics of Transient Intermediates Formed upon UV Excitation of Aromatic Residues. Biophys. J. 2009, 97 (1), 211-226. https://doi.org/10.1016/j.bpj.2009.01.065. 